Carlos Gonzalvo Salas

\section{Arquitecto $y$} doctorando por la ETSA Universitat Rovira i Virgili.
Figura 1. Situación de las centrales nucleares de primera generación. Relación con la topografia, hidrografía y principales áreas habitadas de la península ibérica. Fuente: Elaboración propia.

\title{
La Arquitectura de las Centrales Nucleares en España (1963-1972)
}

Keywords: nuclear power plant, modern architecture, plastic arts, landscape, landmark

This paper explores the Architecture of the first generation nuclear power plants in Spain, developed from 1963 to 1972. The spanish power companies selected to renowned architects such as Antonio Fernández Alba, Ignacio Álvarez Castelao and Antonio Bonet Castellana, for the José Cabrera, Santa María de Garoña and Vandellòs nuclear power plants. The proposals of this group of architects altered the urban structure and incorporate in their proposals plastic disciplines, as sculpture and paintings. Nevertheless, the power companies rejected these proposals because of incompatibilities with safety and nuclear technology. Instead, the three architects performed smaller and budget-scale strategies. These interventions are also required to establish a dialog between the geographic scale of the place, the scale of the nuclear power plant and the human scale of the worker.

$\mathrm{E}^{\mathrm{n}}$ las próximas dos décadas se van a desmantelar más de 400 centrales nucleares en todo el mundo debido al final de su vida útil y por el uso de nuevos métodos para generar electricidad, como por ejemplo, las energías renovables. En España, las centrales nucleares generan más del $20 \%$ de la producción eléctrica ${ }^{1}$. Después de su clausura y descontaminación, sus estructuras internas serán derribadas. Estas construcciones industriales, construidas en España durante los años 60 y 70, contienen la memoria colectiva de toda una generación (figura 1).

Este artículo se enmarca en la investigación sobre la tradición estética de las centrales nucleares de primera generación en España (1963-72). Es necesario apuntar que el tema nuclear ha sido ya estudiado en países como Francia y Estados Unidos, sin em-

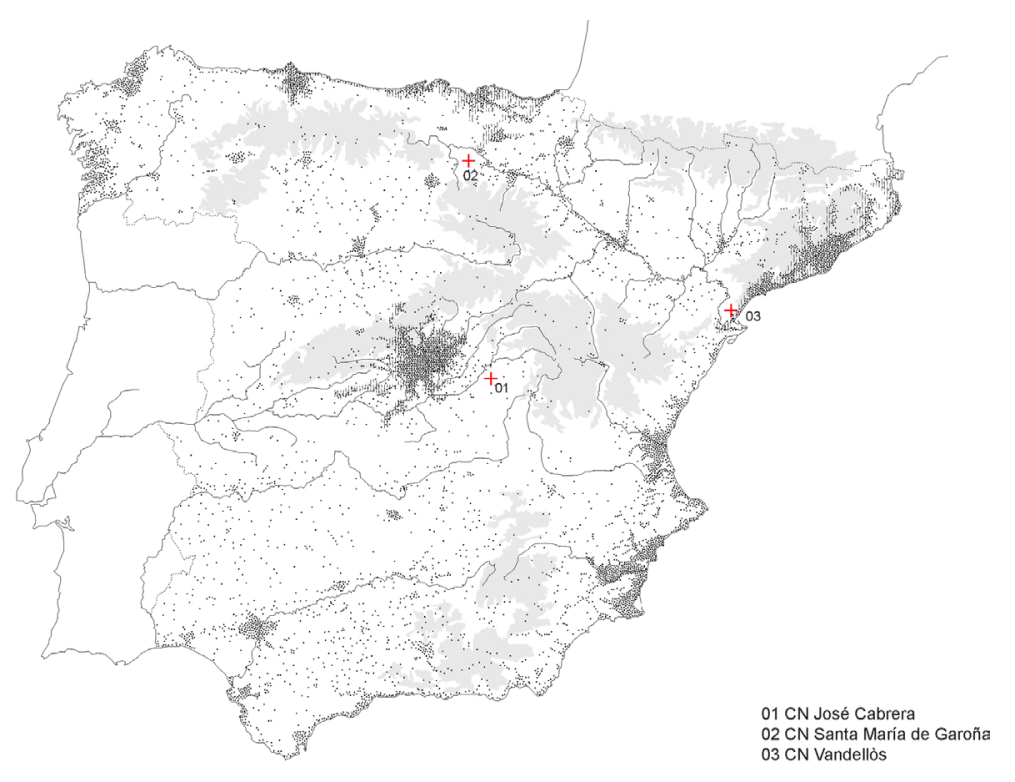

bargo, el papel de la arquitectura en España todavía no ha sido explorado ${ }^{2}$. Arquitectos e ingenieros colaboran conjuntamente en la implantación y construcción de las primeras centrales nucleares: Antonio Fernández Alba (1927), Ignacio Álvarez Castelao (1910-84) y Antonio Bonet Castellana (1913-89), en las centrales nucleares: José Cabrera (1963-68), Santa María de Garoña (1963-71) y Vandellòs (1967-72).

Comúnmente, se denomina arquitectura industrial a fábricas, almacenes, centrales eléctricas de poca potencia y talleres, en las cuales el arquitecto puede actuar con total libertad. Sin embargo, las centrales nucleares tienen una escala geográfica que abarca un basto territorio y requerimientos técnicos superiores a los de cualquier central térmica e hidroeléctrica. La tarea del ingeniero tiene mayor relevancia, como: cálculos de blindajes, distancias de seguridad y estanqueidad. Debido a las limitaciones técnicas - seguridad, disposición de las principales edificaciones, orientación, distancias máximas, dimensiones, formas y materiales - los arquitectos tienen poco margen para intervenir. Mientras que los ingenieros diseñan la forma estructural óptima, los arquitectos diseñan la forma visual, es decir, cómo se perciben estas centrales.

Este artículo denomina arquitectura de las centrales nucleares al trabajo que realizan los arquitectos e ingenieros en relación al lugar, recorrido de aproximación e imagen de estas nuevas centrales nucleares, evitando la definición solamente del objeto como parte de la intervención. Para ello, los tres arquitectos seleccionados por las empresas eléctricas participan en el diseño de las envolventes de 
las edificaciones principales, administrativas, trazado de los accesos y desarrollo de la urbanización. Estas tareas suelen estar asociadas al trabajo de los ingenieros, no obstante, la "preocupación estética" y social que implica la construcción de estas centrales obliga a las empresas eléctricas a cuidar su implantación en enclaves sensibles del territorio, ya sea en la línea de costa o en el curso de un río (Gonzalvo, Ródenas y Zuaznabar 2018:1).

Fernández Alba, con tan solo 7 años de experiencia profesional, es el primer arquitecto seleccionado para colaborar con los ingenieros en la construcción de la CN José Cabrera. Por su parte, Bonet Castellana y Castelao son arquitectos con mayor recorrido profesional. Ambos arquitectos desarrollan actuaciones para la implantación y organización de las principales edificaciones: oficinas, sala de turbinas, vasija del reactor y chimenea. Sus propuestas iniciales modifican la configuración planteada por las empresas eléctricas. No obstante, estas propuestas parecen resultar incompatibles con la seguridad y técnica en este tipo de instalación, por lo que sus propuestas son desestimadas. En su lugar, ambos arquitectos desarrollan actuaciones técnica y económicamente menores en colaboración con el equipo de ingenieros. En este artículo se exploran estas propuestas previas, que por diversos motivos fueron rechazadas: incompatibilidad técnica, criterios de seguridad o problemas en la gestión de las empresas eléctricas.

\section{Implantación de centrales nucleares}

Las centrales nucleares españolas de primera generación utilizan, en esencia, dos modelos opuestos para su implantación en el territorio. Ambos modelos se clasifican según el país de procedencia de la tecnología nuclear: Francia y Estados Unidos. Este último modelo tecnológico aboga por camuflar las centrales nucleares en zonas rurales, alejadas de los principales núcleos habitados. Por el contrario, el modelo francés sitúa estas centrales próximas a las poblaciones, con el cambio de escala que implica la construcción de estos grandes volúmenes de hormigón armado y el impacto visual que supone ${ }^{3}$. Estas dos acciones son antagónicas, mientras que una aboga por pasar desapercibida, la otra utiliza estas construcciones para reivindicar el valor del paisaje.

La Atomic Energy Commission (en adelante, AEC) en Estados Unidos estudia durante las dos primeras décadas la posibilidad de

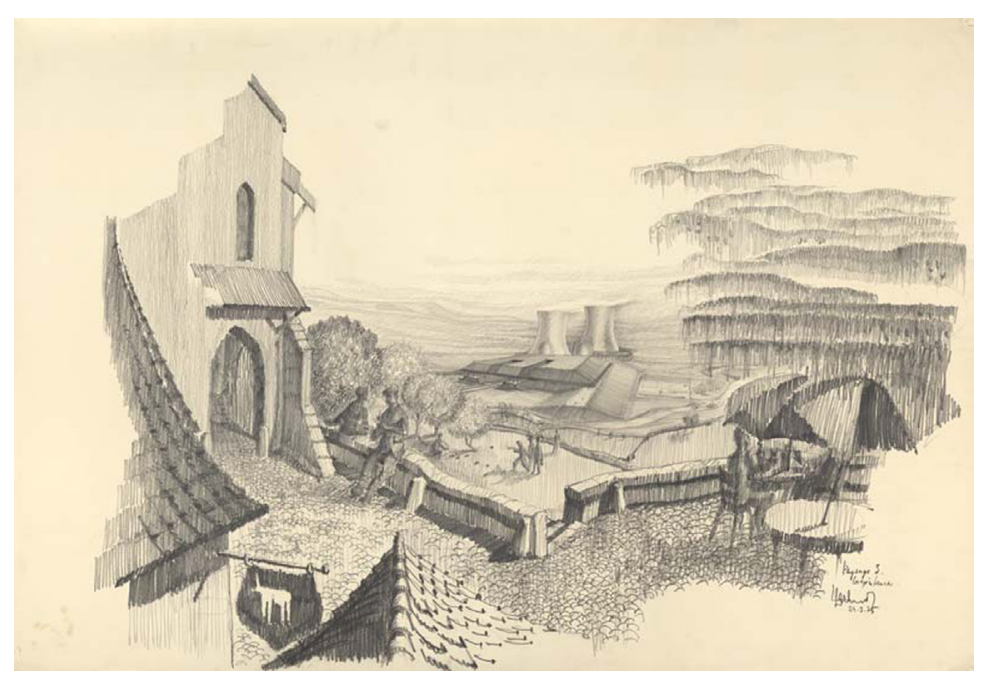

construir estas nuevas centrales nucleares bajo tierra o en alta mar (Yadigarogly y Andersen 1976:110-120). De esta manera, podrian pasar desapercibidas para la población. Una población desinformada y con fobia al uso de este tipo de energía por su utilización militar en la II Guerra Mundial. No obstante, el grado de complejidad técnica y el elevado coste económico para desarrollar estas propuestas impiden su desarrollo. En su lugar, las empresas tecnológicas estadounidenses desarrollan estrategias de camuflaje aprovechando, por un lado, las condiciones del lugar: densidad de población, topografía, clima y vegetación; $y$ por otro lado, estrategias proyectuales: aspectos formales, cromáticos y disposición de las principales edificaciones. Estas estrategias van dirigidas a minimizar el impacto visual y para aumentar la seguridad ante posibles ataques aéreos ${ }^{4}$.

El impacto visual se puede reducir seleccionado lugares donde se pueda utilizar la topografia y los bosques existentes para la ocultación de las estructuras desde los lugares próximos, históricos o recreativos. Restaurar la vegetación e integrar las nuevas estructuras con el entorno pueden mitigar los impactos visuales adversos. (Buchanan 1976:227)

Por su parte, en Francia, se desestima la estrategia de camuflaje y se apuesta por fomentar un mensaje positivo a la población sobre las ventajas de las centrales nucleares. En 1974, en plena crisis del petróleo y boom en la construcción de centrales nucleares, la empresa eléctrica Electricité de France (en adelante, EDF) encomienda al arquitecto Claude Parent ${ }^{5}$ (1923-2016) la coordinación de 12 centrales nucleares. Tras su visita a la central de Fessenheim (1971) entiende el problema que supone colocar gigantescos vo-
Figura 2. Dibujo de Claude Parent. "Les centrales nucléaires. Paysage 3. Coexistence" (1975). Fuente: FRAC Centre, PARE 9975810. 
Figura 3. Imagen de la electricidad (Izquierda) Portada revista Austral 1 (junio 1939) (Centro) Cartel Tennessee Valley Authority (TVA) (1942). Fuente: Department of Energy Oak Ridge (Derecha) Portada revista Arts $\&$ Architecture (marzo 1947). lúmenes en sitios altamente sensibles del territorio, planteando la necesidad de crear un lenguaje arquitectónico específico para centrales nucleares. Este nuevo lenguaje aborda el problema a partir de la reivindicación del paisaje y del papel simbólico de la energía del átomo (figura 2).

\section{La planta crea el sitio. (Bouvier 2005:10)}

En España, la producción eléctrica hasta los años 60 se basa en la energía producida por centrales hidroeléctricas y térmicas. A final de los años 50, la producción de estas centrales es insuficiente debido al aumento de la demanda, sobretodo en las áreas más pobladas e industrializadas: Madrid, País Vasco y Cataluña. El gobierno franquista elabora un plan para la construcción de tres centrales nucleares para aumentar la capacidad de la red eléctrica. Para llevar a cabo su construcción se establece una colaboración internacional. El Gobierno, de la mano del ministro de industria Gregorio López Bravo, importa de Estados Unidos y Francia la tecnología necesaria para la construcción de estas centrales, y con ello, las estrategias para su implantación en el territorio ${ }^{6}$. Las empresas tecnológicas contratadas importan modelos ya experimentados y en funcionamiento en sus paises de origen. Las primeras centrales españolas responden, en esencia, a Big Rock Point (1960-63) y Monticello (1967-71) en Estados Unidos y Saint Laurent des Eaux (1963-69) en Francia (Alonso 2011:12).

En resumen, las centrales nucleares con tecnología estadounidense, CN José Cabrera y CN Santa María de Garona, se ocultan en valles, están mal comunicadas y alejadas de los principales núcleos habitados, con densidades de población que no superan las 0,20 personas por hectárea. Por el contrario, la CN Vandellòs, de tecnología francesa, está próxima a las poblaciones, triplicando los habitantes en sus inmediaciones, está bien comunicada por carretera y ferrocarril y se coloca en un lugar visible. Sin pretensión en su imagen, esta central se convierte en un hito visual que abarca $70 \mathrm{~km}$ en la línea de costa, desde Cabo de Salou hasta el Delta del Ebro.

\section{Encargo y programa}

La electricidad y los elementos que la conforman, como torres y centrales eléctricas, se convierten en objeto propagandístico durante la primera mitad del siglo XX, además se utilizan para representar la modernidad y el progreso tecnológico de la sociedad (figura 3). Para ello, se contrapone con esculturas reconocidas, se utiliza como arma militar durante la II Guerra Mundial y se entiende como parte del progreso tecnológico, del arte y de la arquitectura durante la post guerra. Desde la construcción de la primera central nuclear en 1953, las empresas eléctricas exponen estas nuevas centrales en exposiciones internacionales, como la de Bruselas en 1958, y en los medios de comunicación. Con esta estrategia de comunicación se pretende informar a la sociedad sobre el origen y funcionamiento de estas "Nuevas formas de la Era Atómica". Estas "formas" resultan del cálculo de los ingenieros y están proyectadas desde fuera de las disciplinas estéticas. Mies van der Rohe concluye en el texto "Arquitectura y Tecnología" (1950) que estas "formas" tienen un significado y una forma poderosa, a diferencia de otras construcciones industriales de menor envergadura, en las que el arquitecto puede intervenir con mayor libertad. El objetivo de la arquitectura reside en la convivencia con
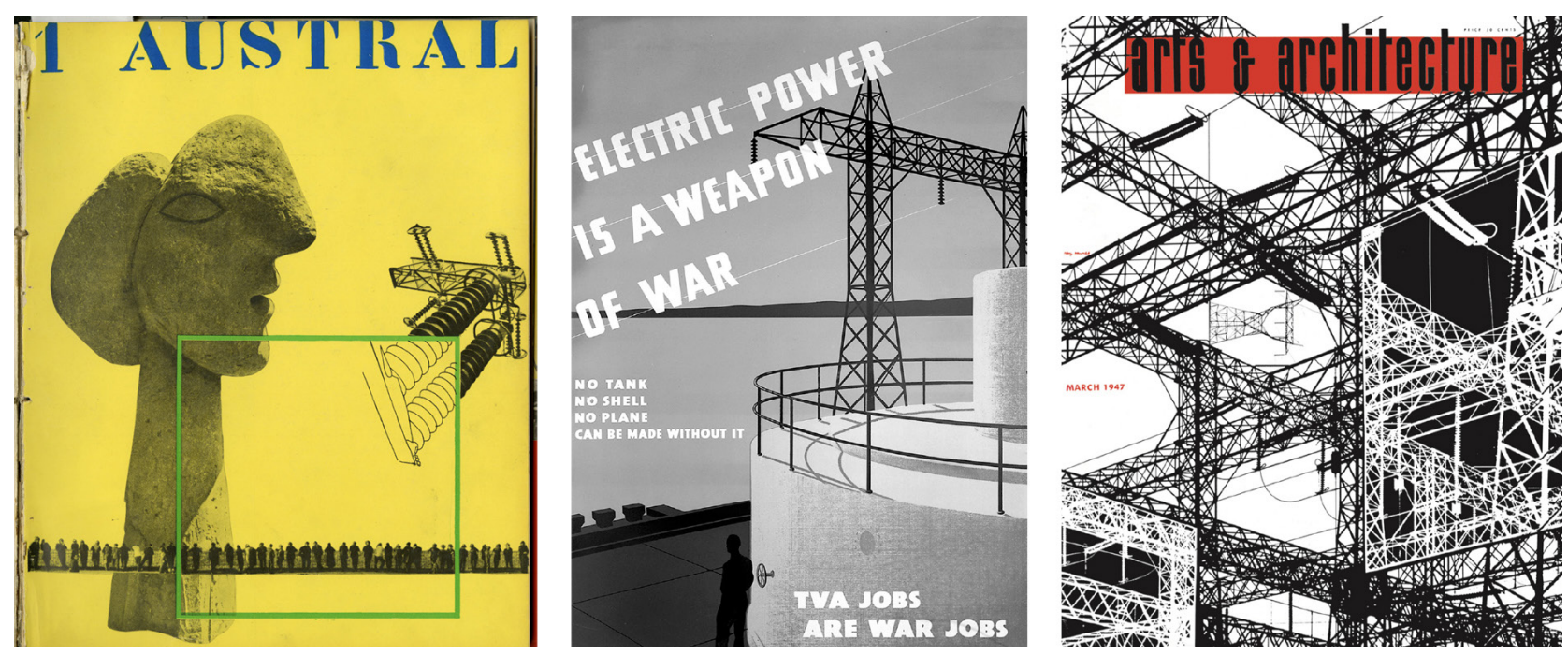
la tecnología, abogando por que una sea la expresión de la otra.

¿Es eso todavía tecnología o es arquitectura?. (Van der Rohe 1950:30)

En este mismo texto, Mies anticipa que ambas disciplinas, arquitectura y tecnología, deben encontrarse al mismo nivel. Si la arquitectura no convive con los progresos tecnológicos que desarrollan los ingenieros, el trabajo de los arquitectos podría pasar a un segundo plano: "algunas personas están convencidas de que la arquitectura será anticuada y reemplazada por la tecnología" (van der Rohe 1950:30). En este sentido, el creciente interés de la arquitectura por estas grandes obras del ingeniero, se puede enmarcar en la repercusión que tuvo la exposición que impulsa el MoMA en 1964: Twentieth Century Engineering (30 de junio - 13 de septiembre). Esta exposición está englobada en el marco de exposiciones del MoMA bajo el título: Art in a Changing World. El catálogo que acompaña la muestra, con prólogo de Arthur Drexler, incluye 140 fotografias en blanco y negro de los 195 proyectos representados.

Esta exposición muestra proyectos de ingeniería en colaboración con arquitectos reconocidos, como: Eduardo Torroja, Félix Candela, Pier Luigi Nervi y Frank Lloyd Wright. El MoMA eleva a categoría artística las grandes obras de ingeniería en colaboración con arquitectos reconocidos, e incluye por primera vez siete centrales nucleares: Marcoule (195559); Dresden (1956-60); Yankee (1957-61); Hunterston (1957-64); San Onafre (1964-68); Connecticut Yankee (1968-72) y Chinon (197784) (figura 4), algunas de ellas realizadas por arquitectos como: J. Demaret; P. Dufau; J. de Mailly; P. Milande o F. Gibberd (Drexler 1969:1-10).

La primera colaboración de un arquitecto con el equipo de ingenieros para la construcción de una central nuclear se realiza en 1954. Hasta entonces, la AEC custodia los documentos relacionados con su construcción. Durante los primeros años, la falta de experiencia del sector de la arquitectura, obliga a las empresas de ingeniería a tomar el control de los proyectos. El papel de los arquitectos se limita a seguir las pautas dictadas por los ingenieros. A finales de los años 50 se publican numerosas monografias sobre temática nuclear dirigidas a arquitectos: AJ Architects en Reino Unido, Werk en Suiza; Progressive Architecture en Estados Unidos; Architecture d'Ajourd'hui en Francia e Informes de la Construcción en España. En estas revistas se pre- sentan las primeras centrales nucleares y se describen las intervenciones que realizan los arquitectos. Como apunta la profesora estadounidense K. Thompson en 1957, el papel de la arquitectura tiene más relevancia conforme pasan los años y, arquitectos y promotores, confian en su trabajo respectivamente.

Cuando se selecciona uno arquitecto; recibe un diagrama de la planta con el que trabajar. Como proyectista, queda poco por definir (...) a menos que tenga el coraje, como algunos han tenido, de probar por si mismo (...) estos edificios resultarán del pensamiento de otros, no de los arquitectos (...) con mayor información, el arquitecto ganará confianza - la suya y la de sus clientes. (Thompson 1957:182)

En este sentido, los primeros reactores nucleares y centros de investigaciones atómicas cuentan con la colaboración de arquitectos reconocidos, como SOM, Estados Unidos (1947-69); Gio Ponti, Brasil (1953); Van der Broek \& Bakema, Holanda (1957-62) y Philip Johnson, Israel (1960) 7 . En España, los promotores de las centrales nucleares, Pedro Durán Farell ${ }^{8}$ y Jaime McVeigh ${ }^{9}$, abogan por cuidar su imagen a partir de una estrecha colaboración entre ingenieros, arquitectos y artistas.

El arquitecto, agobiado por la búsqueda de soluciones técnicas, y falta de un verdadero concepto artístico, se ha separado cada vez más del contacto con las otras artes plásticas, cuya libertad e inquietud se han traducido en una serie escalonada de movimientos, a los que la arquitectura ha sido casi ajena en absoluto. (Bonet, Ferrari-Hardoy; Kurchan 1939:1)

Bonet Castellana publica en junio de 1939, desde el exilio argentino, el primer número de la revista Austral ${ }^{10}$. En el manifiesto

Figura 4. CN Chinon (1977-84). (Demaret y Dufau 1967:32-34).

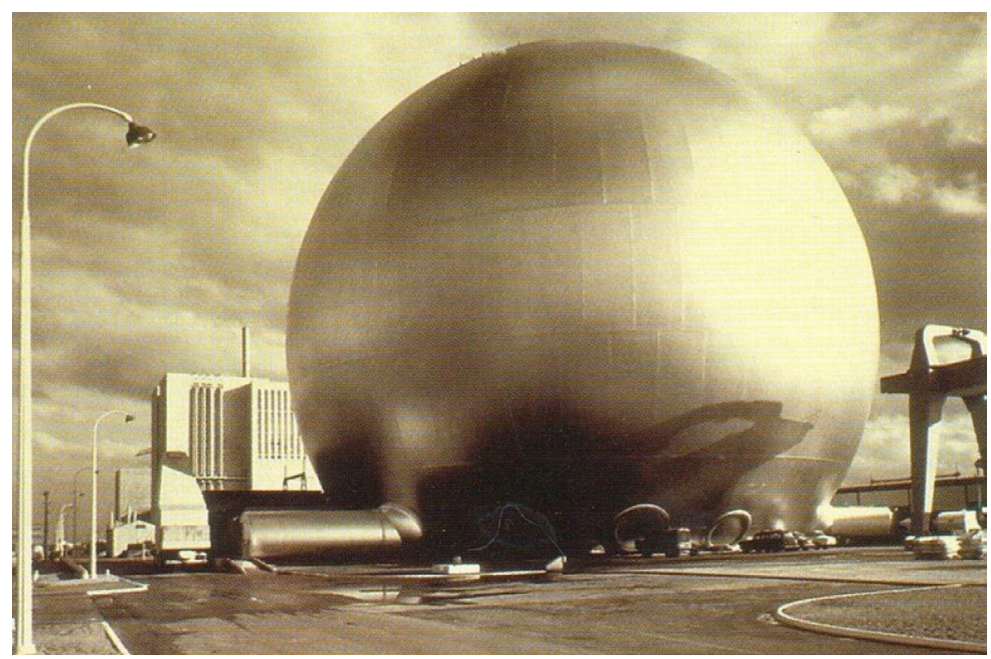




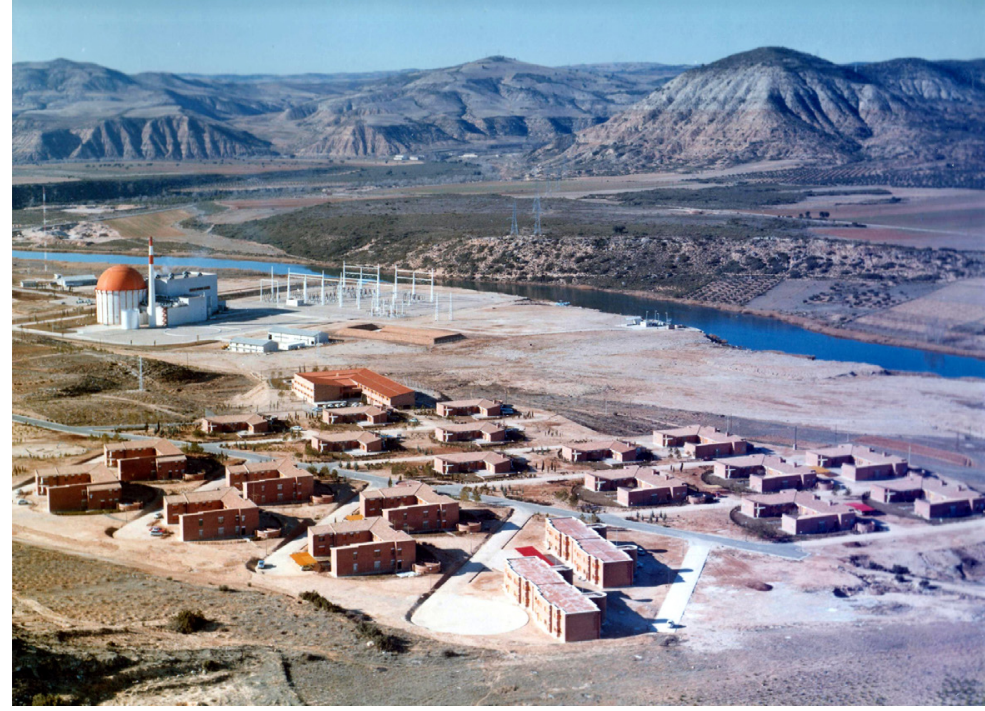

Figura 5. CN José Cabrera. Fotografía aérea. Relación del poblado y la central nuclear (1968). Archivo histórico Fundación Gas Natural.

Figura 6. CN José Cabrera. Trazado urbanístico (versión definitiva). Fuente: Elaboración propia. relata la lejanía de la arquitectura con otras disciplinas plásticas, anticipando conceptualmente la integración de las artes en la sociedad y en la industria. En el mismo texto, aboga por que el arquitecto trabaje conjuntamente con un equipo multidisciplinar, que reúna a ingenieros, constructores, pintores, escultores, entre otras disciplinas artísticas. Estos conceptos, arte y equipo multidisciplinar, se consolidan más adelante en el CIAM de Bridgwater (1947) (Garcia-Pola 1997:93). En la portada de la revista Austral 1, Bonet Castellana contrapone una escultura de $\mathrm{Pa}$ blo Picasso, Cabeza de mujer (1931), con una torre eléctrica (figura 3). Pese no asistir al congreso de 1947, parece anticipar conceptualmente los principios que lo formarian.

Desde los años 50, se incorporan arquitectos para la construcción de centrales eléctricas en España: Corrales y Molezún, Fisac, Vaquero Palacios y Castelao con el pintor del grupo El Paso, Antonio Suárez ${ }^{11}$. Las propuestas que realizan incorporan disciplinas plásticas, a partir de remates escultóricos,

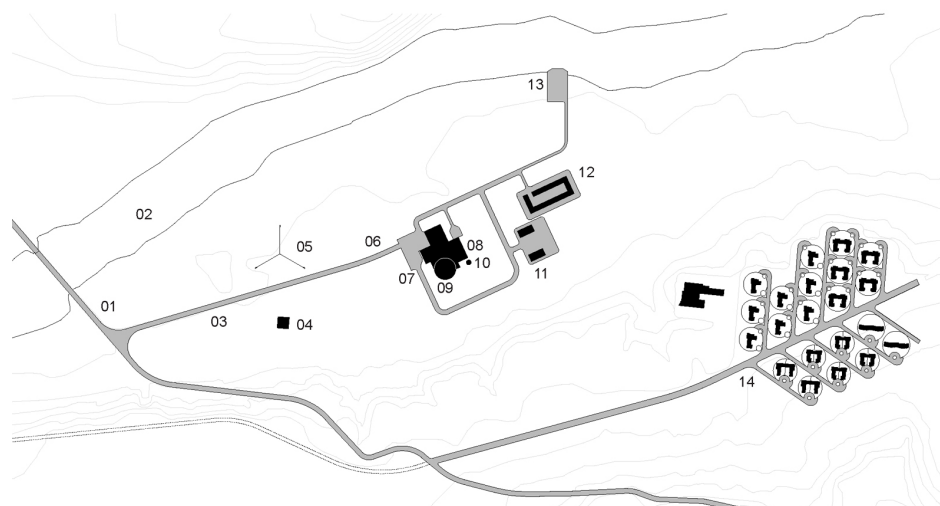

01 Carretera CM-200 02 Río Tajo 03 Viario de acceso 04 Centro de información (no construido) 05 Torre meteorológica 06 Pabellón de control 07 Edificio de oficinas 08 Sala de turbinas 09 Contención del reactor 10 Chimenea 11 Almacenes 12 Almacén de bidones al exterior 13 Toma de agua 14 Poblado para trabajadores

mosaicos, juegos geométricos y orden visual en el recorrido de aproximación. En este sentido, el arquitecto y artista Joaquín Vaquero Palacios es seleccionado por Jaime McVeigh a mediados de los años 60 para "ornamentar" el reactor de la CN José Cabrera. Esta propuesta "engalanaría con adornos" 12 por primera vez una central nuclear. Aunque por motivos internos en la gestión de la empresa eléctrica nunca se llevó a cabo ${ }^{13}$. Vaquero Palacios afirma, igual que Bonet Castellana años antes, que el arte debe formar parte de la sociedad y de la actividad industrial.

Inútil es decir que la integración de las artes no es ningún descubrimiento de ahora. Desde que el hombre puso sus pies sobre la tierra y hubo de guarecerse al cobijo de algo, la integración con la pintura y la escultura ha tenido lugar. El fenómeno continúa sin detenerse, ha venido la industria y hoy la integración es una absoluta necesidad ¿por qué? ¿para qué? Pues porque nuestra actividad actual está desbordada y nuestro organismo necesita ser apaciguado de alguna forma para sobrevivir a la tensión a la que se somete cada vez con mayor exigencia. (Vaquero 1998:116)

\section{Central Nuclear José Cabrera (1963-1968)}

La empresa eléctrica Unión Eléctrica Madrileña (en adelante, UEM) construye la primera central nuclear española en las proximidades de Madrid en 1963. Fernández Alba participa en el diseño del pabellón de control y centro de información, así como en la envolvente del reactor, sala de turbinas y oficinas. El encargo se complementa con la construcción de un poblado para alojar a 58 trabajadores en la parcela colindante y una residencia para 26 trabajadores temporales (figura 5).

La central nuclear se sitúa en el margen izquierdo del río Tajo a su paso por Almonacid de Zorita, provincia de Guadalajara. Esta central, la primera de tecnología estadounidense en España, abastece al mercado de Madrid, situándose en una zona rural suficientemente despoblada, alejada de los núcleos habitados: $70 \mathrm{~km}$ al este de la capital y $45 \mathrm{~km}$ de Guadalajara. La parcela, de topografia plana y 20 ha de extensión, limita en el lado norte con el cauce del río, en el lado sur con el poblado para trabajadores, en el lado este con una zona de huertos y en el lado oeste con la carretera CM-200 que le da acceso desde Pastrana y Almonacid de Zorita.

La central (figura 6), siguiendo los protocolos establecidos por la AEC, se ubica en una 
zona geográfica donde la topografia del valle permite ocultar la construcción. Pese el gran tamaño de la intervención, $90 \mathrm{~m}$ de largo, 60 $\mathrm{m}$ de ancho y $70 \mathrm{~m}$ de alto, el primer contacto visual se produce a $1 \mathrm{~km}$ de distancia desde la carretera de acceso. Sobre un terreno árido con escasa vegetación emerge una cúpula metálica brillante de color naranja, de 33 $\mathrm{m}$ de diámetro y $40 \mathrm{~m}$ de altura. Fernández Alba describe el entorno como un territorio de extrema dureza por su clima árido y escasas precipitaciones. Se plantea la hipótesis de que a partir de este factor de emplazamiento se decante la utilización del naranja como color predominante de la propuesta para "camuflarse" en el entorno ${ }^{14}$.

La central se coloca en paralelo a la carretera que le da acceso, con una separación de $530 \mathrm{~m}$. La configuración de los elementos principales se disponen de tal manera que las partes más altas, chimenea y vasija del reactor, están próximas a la ladera del lado sur; y sala de turbinas y oficinas, de menor altura, próximas al cauce del río. Debido a su colocación en relación al trazado de la carretera, el conjunto queda expuesto tras atravesar el umbral de acceso que supone el puente sobre el río desde el lado norte (figura 7). Tras realizar un giro de $90^{\circ}$ en el recorrido de acceso, se encara frontalmente la fachada principal del conjunto, orientada a oeste. Esta fachada contiene la totalidad de edificaciones: centro de información; pabellón de control; oficinas; sala de turbinas; vasija del reactor y chimenea. En el lado superior derecho se sitúa el poblado para trabajadores, construido sobre una plataforma artificial.

Esta fachada principal de acceso genera una zona residual situada justo detrás, la fachada trasera, donde se prevé en los años 70 la construcción de la fase 2 de la central nuclear. En este lado de la parcela, con una correcta iluminación hasta mediodía, se disponen los almacenes y depósitos de bidones al exterior. Es necesario apuntar, que esta fachada es la principal para los trabajadores que están alojados en el poblado, $28 \mathrm{~m}$ por encima de la cota de la central (figura 8).

Fernández Alba no proyecta las edificaciones principales, tan solo proyecta sus envolventes. Las diferentes versiones que se conservan en su archivo profesional van dirigidas a minimizar el impacto visual (figura 9). Fernández Alba desarrolla una misma solución agrupando el edificio administrativo, de 23 x 18 m y 14 m de altura, con la sala de turbinas, de 46 × $28 \mathrm{~m}$ y $27 \mathrm{~m}$ de altura. Para ello, subraya la estructura vertical de pilares,

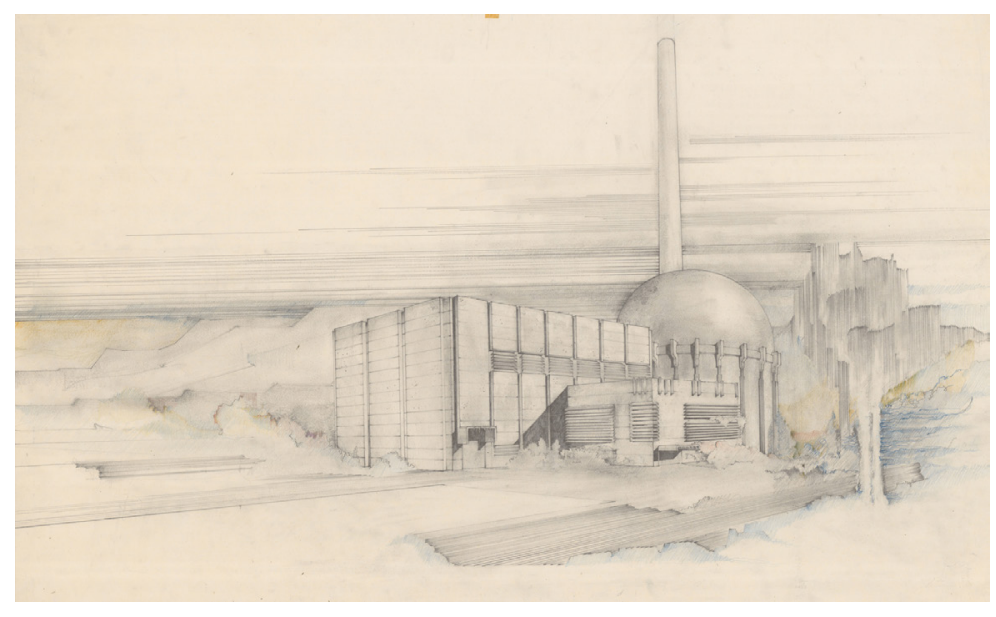

separados cada 6,50 m. Al mismo tiempo, agrupa los huecos colocando unas lamas metálicas en posición horizontal de color naranja. En la vasija del reactor, justo al lado de la sala de turbinas, opera de la misma manera: aprovecha una necesidad, como es desaguar la cúpula, para la colocación de 24 bajantes metálicos, de $30 \mathrm{~cm}$ de diámetro y $22 \mathrm{~m}$ de altura. Estos bajantes están colocados cada $15^{\circ}$ y se adosan al muro cilindrico construido con hormigón fuertemente armado de $1 \mathrm{~m}$ de espesor. Sin ninguna pretensión, estos bajantes, reducen el impacto visual que podria tener el cilindro de hormigón sin "ornamento", generando planos consecutivos de 4,30 x 22 $\mathrm{m}$ en torno al cilindro de la vasija del reactor.

\section{Central Nuclear Santa Maria de Garoña (1963-1970)}

La empresa eléctrica Nucleares del Norte (en adelante, Nuclenor), de la que forma par-

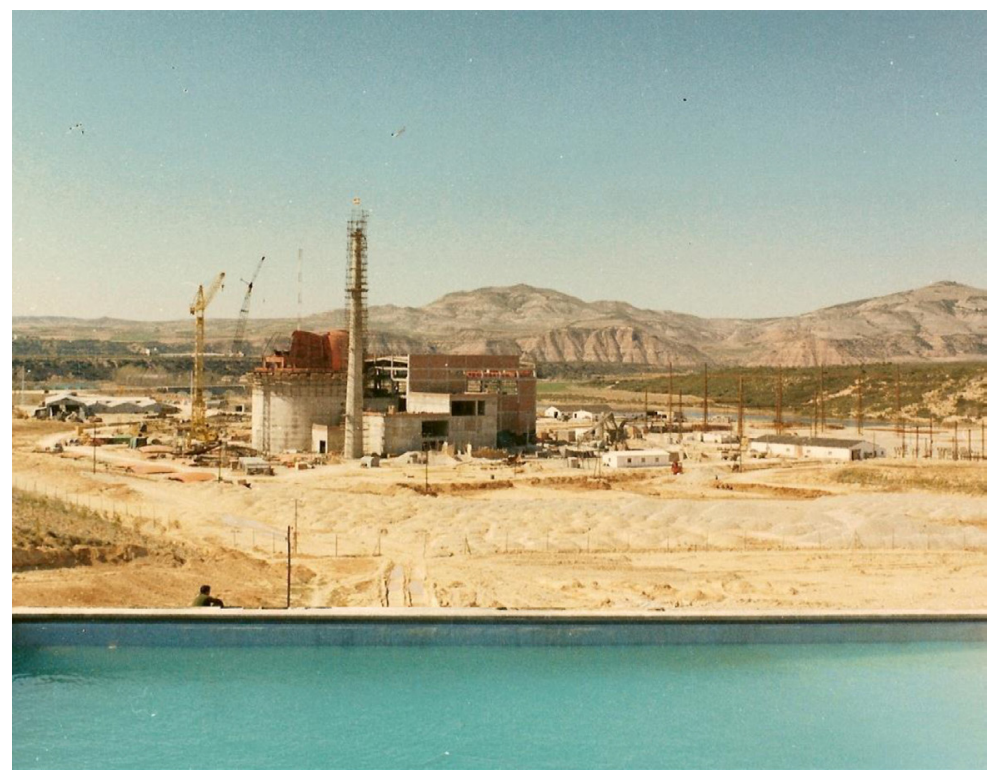

Figura 7. CN José Cabrera. Perspectiva del conjunto (ca. 1966). Fuente: Servicio Histórico COAM, fondo Antonio Fernández Alba.

Figura 8. CN José Cabrera. Fotografia de la central nuclear desde la piscina del poblado durante la construcción (ca. 1966). Fuente: Archivo Histórico Fundación Gas Natural. 
Figura 9. CN José Cabrera. Alzado principal desde el acceso. Fuente: Elaboración propia.
Figura 10. CN Santa María de Garoña. Fotografía desde el embalse de Sobrón (1971). (VV. AA 1971:851)

Figura 11. CN Santa María de Garoña. Trazado urbanístico (primera versión). Fuente: Elaboración propia.

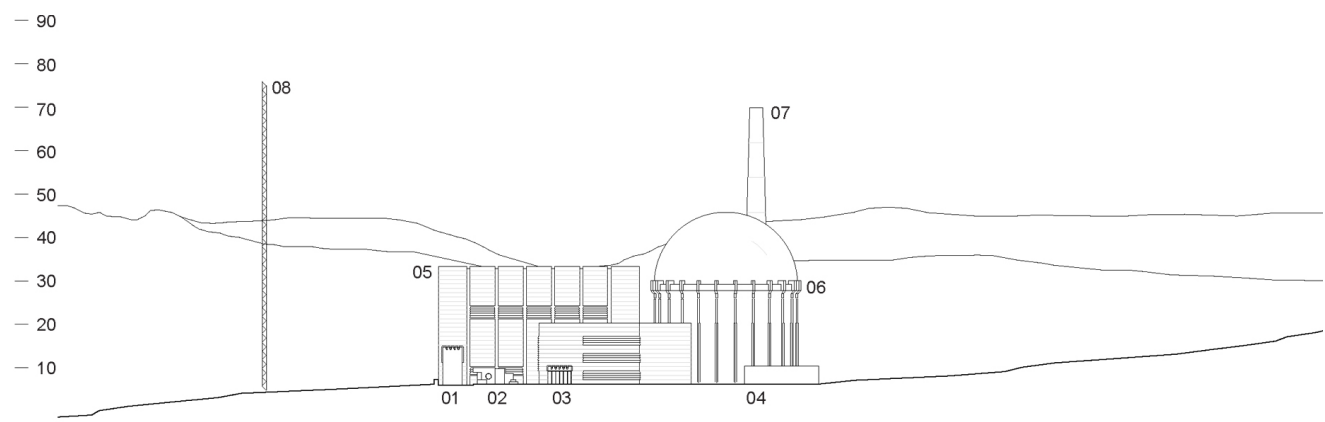

01 Acceso por carretera 02 Pabellón de control 03 Edificio administrativo 04 Centro de información 05 Sala de turbinas 06 Edificio de contención de la vasija del reactor 07 Chimenea de refrigeración 08 Estación meteorológica

te Electra de Viesgo e Iberduero, construye la segunda central nuclear española en la provincia de Burgos en 1963. Castelao colabora con Juan José Elorza y el equipo de ingenieros de esta empresa eléctrica desde 1948 para la construcción de 4 centrales hidroeléctricas y 13 poblados para trabajadores. El programa de esta central se complementa con un poblado para alojar a los altos cargos y una residencia para trabajadores temporales, ubicada en la orilla opuesta de la central, a escasos $600 \mathrm{~m}$. Las categorias profesionales de menor rango se alojan en un poblado construido a $30 \mathrm{~km}$ al este, en la periferia de Miranda de Ebro $^{16}$.

La central nuclear se implanta en un meandro en el margen izquierdo del río Ebro a su paso por Santa María de Garoña (figura 10). Esta central abastece principalmente a la industria de la costa cantábrica y País Vasco.
Se sitúa en una zona rural despoblada, alejada de los principales núcleos habitados: 61 $\mathrm{km}$ al sur de Bilbao y $89 \mathrm{~km}$ de Santander. La parcela, de 61 ha de extensión y de topografia plana, limita en los lados norte y oeste con el cauce del río, en el este con el embalse de Sobrón y en el lado sur con una zona de huertos.

Una agradable visión del conjunto en el que hemos acusado el juego de volúmenes, planos $y$ sombras. ${ }^{17}$

Con esta cita, define Castelao la primera versión de la central en julio de $1965^{18}$. La solución que plantea es compacta y agrupa los volúmenes de sala de turbinas, vasija del reactor, chimenea y oficinas. Con su intervención ocupa la mínima superficie de la parcela y genera una percepción dinámica desde la carretera (figura 11). Esta propuesta toma

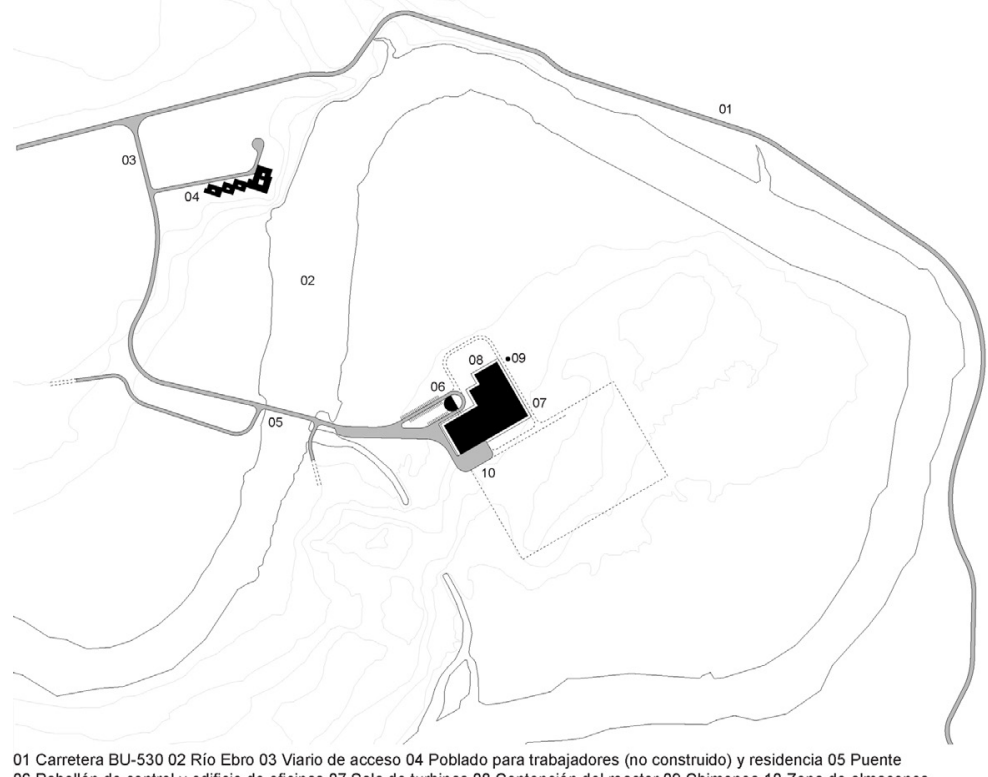

01 Carretera BU-530 02 Río Ebro 03 Viario de acceso 04 Poblado para trabajadores (no construido) y residencia 05 Puente
06 Pabellón de control y edificio de oficinas 07 Sala de turbinas 08 Contención del reactor 09 Chimenea 10 Zona de almacenes

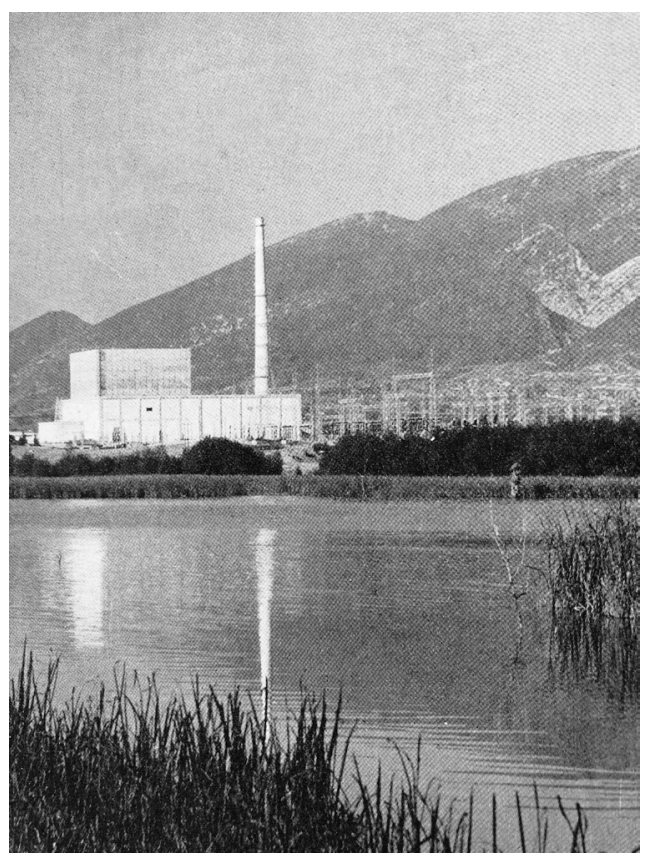


como referencia la central nuclear de Monticello en Estados Unidos (1966) (Alonso 2011:12). Castelao modifica la configuración inicial de las edificaciones principales para establecer un orden visual en el descubrimiento, acentuándolos a través de juegos de luces y sombras. Además, como en el proyecto industrial de La Casera, Oviedo (1968) recurre al efecto propagandístico para resolver la ordenación. Una composición armoniosa de los volúmenes y materiales podría tener un efecto positivo en los trabajadores y la sociedad.

(...) la propaganda que el conjunto industrial ha de facilitar por su efecto sobre el transeúnte, futuro consumido (...) ordenación adecuada de las diferentes partes, y efecto propagandistico de su conjunto exterior (...) fue pensado en relación con el punto de vista principal. $^{19}$

Castelao dicta con precisión el ritual de aproximación. En este sentido, este recorrido comienza en la carretera que da acceso desde Miranda. Ésta transcurre por el lado opuesto a la central, siguiendo la llanura del valle, en paralelo al cauce del río. Castelao, como realiza en las centrales hidroeléctricas de Silvón, Arbón y Las Arenas, reconoce la escala geográfica y la utiliza en beneficio del proyecto, dilatando en tiempo y distancia el momento exacto del descubrimiento.

Siguiendo los principios establecidos en Estados Unidos, la central nuclear, igual que la CN José Cabrera, de tecnología estadounidense, se oculta en el valle. El primer contacto visual se produce desde la carretera de acceso a $5 \mathrm{~km}$ al este, dirección Miranda de Ebro. Este tramo cuenta con 5 túneles y una longitud aproximada de $410 \mathrm{~m}$. Tras atravesar el último túnel, de $150 \mathrm{~m}$ de longitud, aparece en la distancia la silueta de una chimenea que emerge $90 \mathrm{~m}$ sobre una masa de chopos. A partir de este punto, se recorre 3,50 $\mathrm{km}$ junto al embalse en el lado izquierdo, un desmonte en el derecho y en el punto de vista la chimenea.

Una vez atravesado este tramo, se llega al meandro donde está situada la central. En este punto del recorrido, tan solo se muestra la chimenea y se insinúan ciertas partes de los grandes volúmenes de hormigón macizo a través de la vegetación estratégicamente plantada (figura 12 $2^{20}$. La chimenea, ubicada en el centro del meandro, se convierte en un elemento de referencia que acompaña en el lado izquierdo durante el último tramo hasta el cruce de acceso. Este cruce se sitúa $15 \mathrm{~m}$ por encima de la cota de la central. De esta

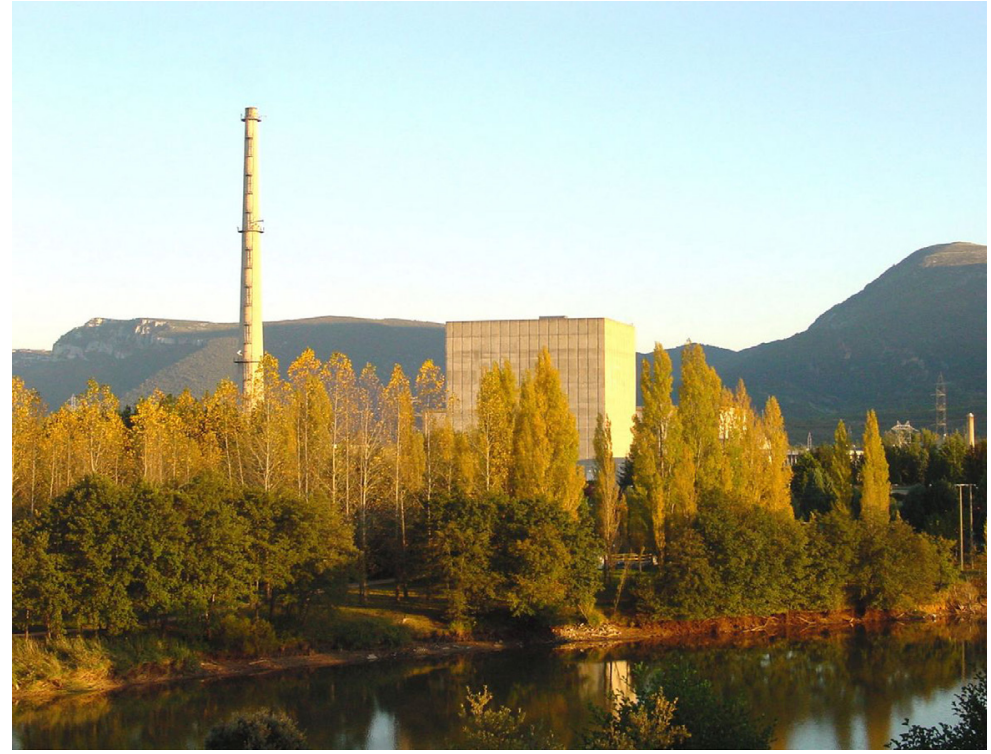

manera, cuando se toma el desvio, se tiene un control visual sobre el lugar de trabajo. A partir de este punto, comienza un camino de descenso de $875 \mathrm{~m}$ de longitud hasta llegar a un puente para cruzar el río.

Este puente, construido en 1964 sobre una antigua presa, sin pretensión programática, se convierte en un umbral de acceso. Tras atravesarlo, el personal está "dentro del recinto protegido". Pese a las recomendaciones fijadas por la AEC años antes frente la seguridad de este tipo de instalaciones, la propuesta de Castelao no contempla una cerca perimetral. En su lugar, construye un edificio de control situado frente al acceso a una cota elevada, que permite tener un control visual desde el cruce de acceso hasta el puente sobre el río (figura 13).

Figura 12. CN Santa María de Garoña. Fotografía desde la carretera (2016). Fuente: Archivo Nuclenor.

Figura 13. CN Santa María de Garoña. Fotografía desde el puente de acceso. Fuente: Archivo Nuclenor.

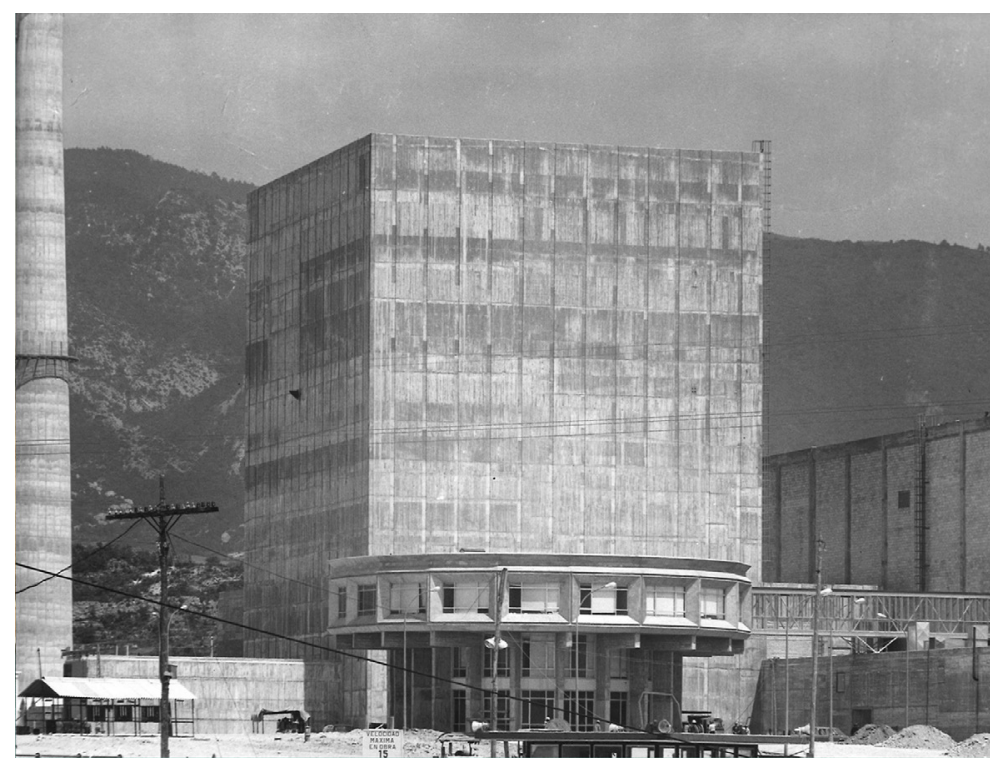


Figura 14. CN Santa María de Garoña. Alzado principal desde el acceso. Fuente: Elaboración propia.
Figura 15. CN Santa Maria de Garoña. Fotografía desde Santa María de Garoña (ca. 1970). Fuente: Department of Energy US.

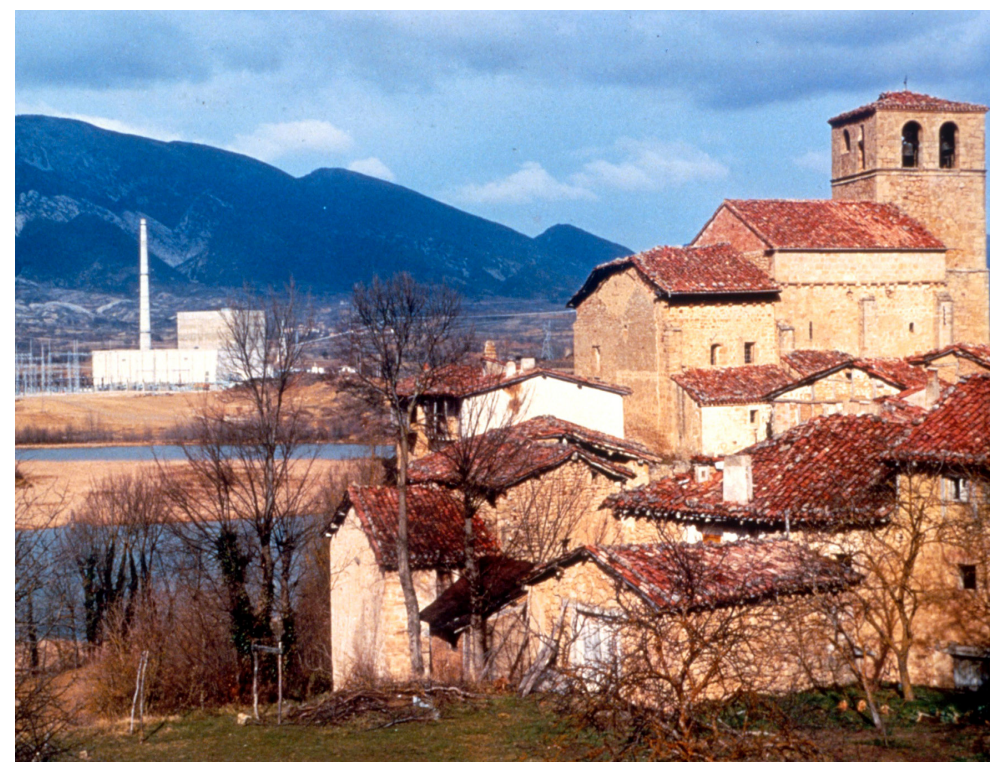

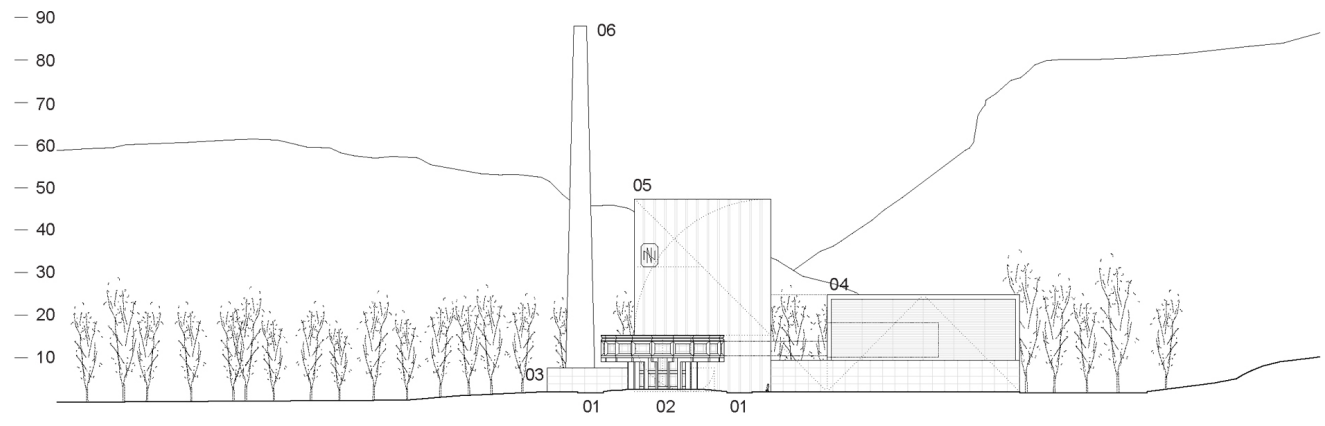

01 Acceso por carretera 02 Pabellón de control y edificio administrativo 03 Zócalo auxiliar y de servicios 04 Sala de turbinas 05 Edificio de contención de la vasija del reactor 06 Chimenea de refrigeración

En este punto del recorrido, ya se ha bordeado la central desde dos costados y cruzado el puente observando el tercero. El conjunto que forma el reactor, sala de turbinas y oficinas está girado $45^{\circ}$ respecto la orientación del puente y $5 \mathrm{~m}$ por encima, provocando que se realice un último giro para encauzar la mirada a la fachada principal, orienta a suroeste. Desde este punto de vista, se muestra toda la intervención en una sola captura. La chimenea, que nos ha facilitado la aproximación, queda oculta tras el reactor. Esta fachada está a contraluz durante toda la mañana y totalmente iluminada a partir de mediodía. La imagen que provoca esta central está intencionada; en primer lugar, por las características del emplazamiento, seguido por la composición de los volúmenes en relación al entorno.

Castelao reconoce las características fisicas del lugar: la planicie de un valle, con montañas de gran altura al fondo y el cauce del río Ebro en primer plano. Este trabajo se complementa con la composición de los volú- menes y cómo éstos se perciben desde el exterior. En este caso, desde el viario de acceso, Santa Maria de Garoña (figura 15) y desde el poblado para trabajadores. La disposición de los volúmenes, a diferencia de la CN José Cabrera, deja entrever la parte posterior, fragmentando la escala y evitando que la intervención sea una barrera visual de grandes dimensiones impuesta en el paisaje del valle.

La propuesta de Castelao incluye una segregación de circulaciones. Por un lado, la red peatonal se resuelve mediante un paso elevado, que conecta las oficinas con la sala de turbinas; y pasos pavimentados en el plano del suelo que bordean las edificaciones, formados con piezas prefabricadas de hormigón. Los accesos del personal están situados próximos a estos pasos peatonales. Por otro lado, el trazado rodado está jerarquizado en función del tipo de vehículo: automóvil y maquinaria. El viario para estos últimos tiene un ancho de $10 \mathrm{~m}$ y está limitado a las zonas de carga y descarga, situadas en la parte sur y este de la parcela; el de automóviles, de $6 \mathrm{~m}$ de ancho, queda limitado hasta el edificio de control y administración. Este edificio actúa como una rótula del viario facilitando el control y la entrada y salida de vehículos.

$\mathrm{Si}$ analizamos el alzado principal (figura 14), el conjunto de la central cuenta con cinco elementos divisibles compuestos de manera armoniosa: vasija del reactor, sala de turbinas, chimenea, edificio administrativo y un zócalo que conecta física y visualmente estos elementos. El alzado de la vasija del reactor tiene una proporción áurea, de $32 \mathrm{~m}$ de ancho y $46 \mathrm{~m}$ de altura. Mientras que el basamento, donde se encuentran las oficinas de $13 \mathrm{~m}$ de altura, es la parte proporcional; la parte que resta por encima es un cuadrado de $32 \mathrm{~m}$ de lado. Los muros de contención del reactor son de hormigón visto de $1 \mathrm{~m}$ de espesor, marcando el despiece vertical del encofrado cada $2,50 \mathrm{~m}$. En este lado del reactor se sitúa la 


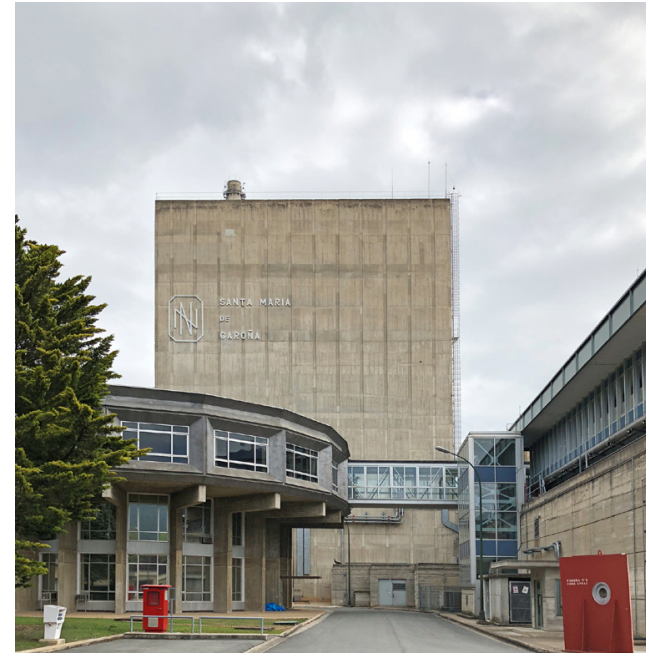

marca de Nuclenor. Este elemento corporativo está situado en el lado izquierdo a dos tercios de la base (figura 16).

La sala de turbinas está situada a la derecha de la vasija. Entre ésta y el reactor se dispone de una separación de $13 \mathrm{~m}$, distancia que resulta de la proyección de la diagonal del cuadrado del reactor en contacto con el plano del suelo. La sala de turbinas tiene un ancho de $45 \mathrm{~m}$ y una altura de $23 \mathrm{~m}$. En esta edificación la estructura de hormigón de pilares y jácenas, de $1 \mathrm{~m}$ de canto, queda vista. Los paños que cierran perimetralmente son de ladrillo blanco rehundido $5 \mathrm{~cm}$, dejando visible una línea de sombra que varía a lo largo del día. Esta sala, debido a los requerimientos técnicos frente la radioactividad, a diferencia de la sala de turbinas de la central anterior, no puede tener ninguna abertura al exterior (figura 17).

La chimenea tiene $90 \mathrm{~m}$ de altura y un diámetro variable, de $7 \mathrm{~m}$ en la base y $3 \mathrm{~m}$ en la parte superior. La chimenea se sitúa en lado izquierdo de la composición y compensa visualmente el peso de la sala de turbinas en el lado derecho. Este ejercicio compositivo se remata con la construcción de un zócalo de hormigón macizo que vincula física y visualmente las piezas anteriormente estudiadas, de 7,20 m de altura. Este zócalo es la construcción más próxima a la circulación del personal. El hormigón cuenta con un despiece de $3 \times 1,8 \mathrm{~m}$, disminuyendo la escala y adaptándose a la medida de los trabajadores.

\section{Central Nuclear Vandellòs (1967-1972)}

En octubre de 1964, el ministro francés de investigación y asuntos nucleares, Gaston Palewski, visita al ministro de industria Gregorio López Bravo con el objetivo de ini- ciar conversaciones para construir la tercera central nuclear, ésta de tecnología francesa de la empresa EDF. En 1966, ya se dispone de un informe completo sobre el esquema de la operación con la elección del lugar y la estructura empresarial que lleva a cabo la construcción y explotación. Al mismo tiempo, se construye el poblado Hifrensa (Hispano Francesa de Energía Nuclear, S.A.). Se trata de un conjunto residencial formado por la agrupación de viviendas para alojar a los trabajadores de la central. Cuenta con equipamientos a escala de barrio y dotaciones de infraestructura para abastecer a las 280 viviendas con agua potable, electricidad y gas propano. Bonet Castellana recibe el encargo del poblado en 1967, así como la realización de las dependencias administrativas, obra civil y otros edificios técnicos en el emplazamiento de la central.

La central nuclear se implanta en la vertiente sur del Coll de Balaguer (figuras $18 \mathrm{y}$ 19), un lugar estratégico desde la antigüedad, por la dificultad de paso debido al estrangulamiento que forman las montañas que se levantan en la misma línea de costa, superando los $700 \mathrm{~m}$ de altura. El Coll de Balaguer actúa de rótula entre el poblado, en el lado norte y la central, en el lado sur. Ambos emplazamientos distan $6 \mathrm{~km}$ y están comunicados por la carretera nacional N-340. La central nuclear abastece de electricidad a la región catalana y sur de Francia. Debido al cambio de modelo tecnológico, la central de Vandellòs se construye próxima a las poblaciones y cuenta con mejoras en la red de comunicación. Se sitúa a unos $40 \mathrm{~km}$ al sur de Tarragona y $110 \mathrm{~km}$ de Barcelona. La parcela tiene una extensión de 25 ha y se organiza a partir de plataformas hasta la cota del mar. Los límites de la parcela en el sentido perpendicular mar-montaña son dos barrancos, Gestell y Llèria; en el

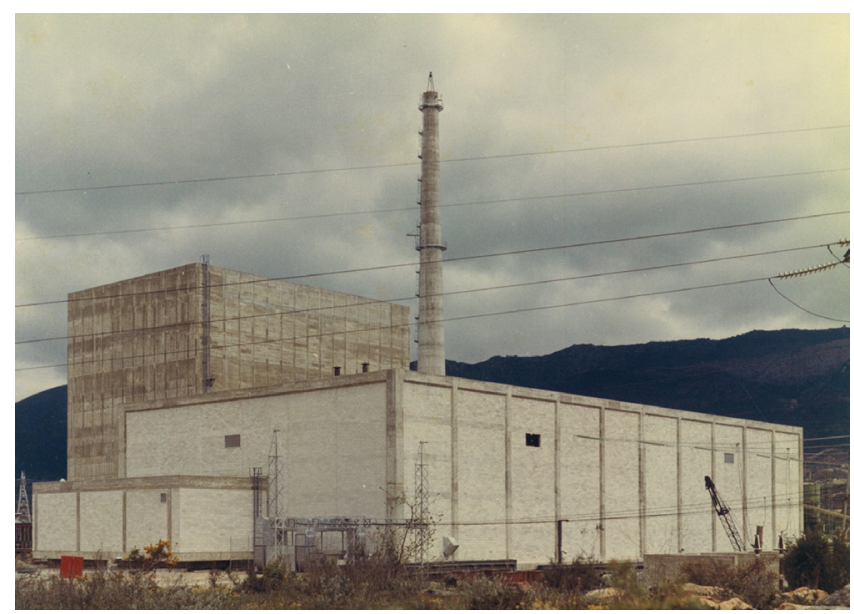

Figura 16. CN Santa María de Garoña. Fotografía del acceso (mayo 2018). Fuente: Fotografía del autor.

Figura 17. CN Santa María de Garoña. Relación entre las principales edificaciones (ca. 1970). Fuente: Archivo Nuclenor. 
Figura 18. CN Vandellòs. Fotografía desde la playa del Arenal de l'Hospitalet de l'Infant (ca. 1972). Fuente: Arxiu Municipal de Vandellòs i l'Hospitalet de l'Infant.

Figura 19. CN Vandellòs. Fotografía desde la playa del Arenal de l'Hospitalet de l'Infant (1970). Fuente: Arxiu Municipal de Vandellòs i l'Hospitalet de l'Infant.

Figura 20. CN Vandellòs. Trazado urbanístico (primera versión). Fuente: Elaboración propia.
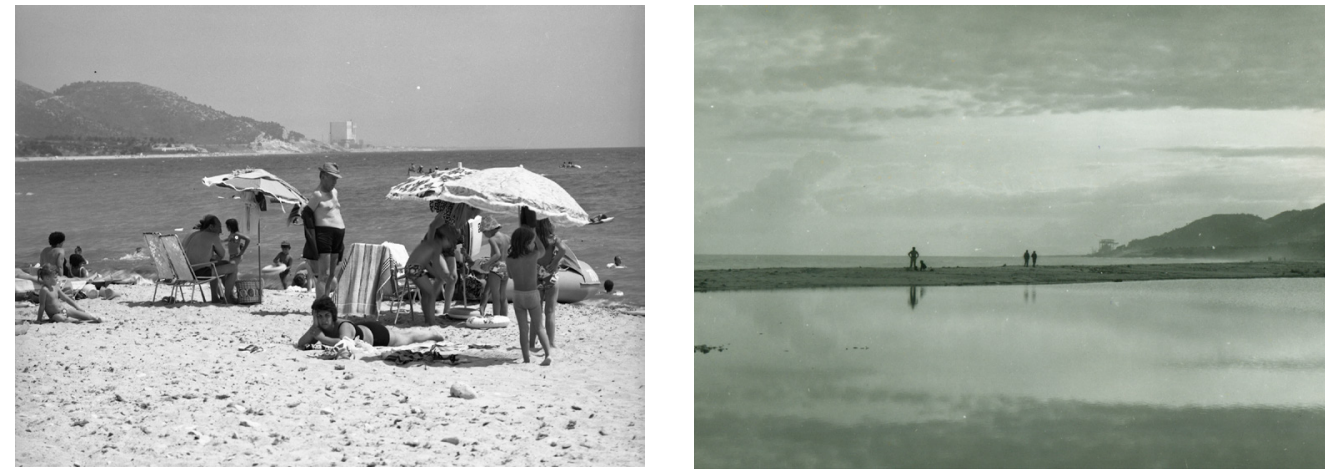

lado sur limita con el mar Mediterráneo y en el lado norte con la cordillera prelitoral, la vía férrea Barcelona-Valencia y la carretera que le da acceso desde el poblado Hifrensa.

A diferencia de Fernández Alba y Castelao, Bonet Castellana participa en el debate urbanístico y artístico europeo. Esta condición le permite incorporar en su obra la experimentación, tanto con las artes plásticas, como mejoras en los trazados urbanísticos. En este sentido, tanto Bonet Castellana en la CN Vandellòs, como Claude Parent en las centrales nucleares francesas, ambos arquitectos antiguos colaboradores de Le Corbusier, desarrollan propuestas para una coexistencia de estas centrales nucleares en paisajes totalmente virgenes. Frente el modelo estadounidense, ambos arquitectos reivindican el papel simbólico de la energía del átomo y del paisaje a partir de su activación con gigantescas estructuras. Parent defiende la idea de paisaje "arquitectónico" a partir de la resolución de tres escalas: territorial, intermedia y próxima (Parent 1989:5). Los estudios que lleva a cabo en Francia se enfocan a la estandarización de las centrales nucleares a partir de resolver armoniosamente la relación de los principales elementos, entre ellos y con el entorno.

Bonet Castellana recibe el esquema de funcionamiento de la central, realizado por $\mathrm{EDF}$, en 1967. En este esquema se indica con precisión la ubicación de las principales edificaciones y del sistema viario. La propuesta tiene limitaciones técnicas: topografia, posición de las edificaciones, distancias máximas, orientación y materiales.

La propuesta de EDF organiza el conjunto en tres plataformas descendentes: $+20,+16$ $\mathrm{y}+9 \mathrm{~m}$. El viario que conecta el acceso con todas las edificaciones está ubicado en paralelo a la línea de costa y da la espalda al mar. En cuanto a las edificaciones principales, se disponen de tal manera que, en la plataforma más elevada, se construyen las de menor entidad, como talleres y depósitos; y en la más baja, las edificaciones más altas, como la vasija del reactor y la central eléctrica auxiliar. A partir de esta organización, se minimiza el impacto visual desde el viario de acceso.

Debido a la condición topográfica del entorno y del trazado sinuoso de la carretera que da acceso a la central desde el poblado para trabajadores, el primer contacto visual se produce a $1 \mathrm{~km}$ de distancia, dirección norte. En este caso, se produce la misma situación que en la CN Santa María de Garoña. El desvío de

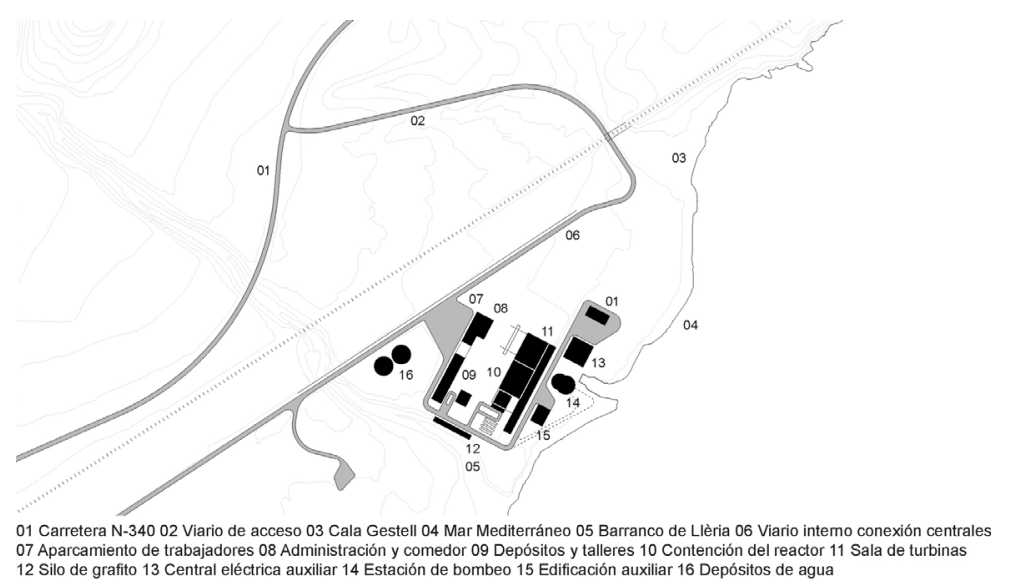
la carretera de acceso a la central se sitúa en una cota $20 \mathrm{~m}$ por encima de la central. En este punto comienza un camino de descenso de $665 \mathrm{~m}$ de longitud, hasta la plataforma de la cota +16 . En esta plataforma se encuentra un puente, construido en la segunda mitad del siglo XIX, para atravesar la vía del tren. Este puente, de más de $20 \mathrm{~m}$ de altura delimita visualmente el área restringida de la central. Tras atravesarlo, se descubre el mar, junto con la Cala Gestell en el lado izquierdo y la central nuclear en el lado derecho.

Bonet Castellana, a diferencia de la propuesta de los ingenieros de EDF, plantea una entrada por detrás (figura 20), que permita descubrir el lugar a través del mar. Con esta 


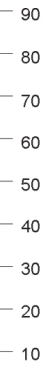

10

01 acceso por carretera 02 porche de acceso 03 edificio administrativo y de control 04 comedor 05 depósitos y talleres 06 sala de turbinas (enterrada) 07 puente grúa 08 edificio de contención de la vasija del reactor 09 sala de control

propuesta, además de evitar darle la espalda a la línea de costa, se reduce considerablemente el viario a lo estrictamente necesario. Este acceso trasero utiliza un viario secundario planteado por EDF en el lado norte de la parcela, en contacto con el talud de la vía ferroviaria. Este viario permitiria comunicar la central con las posibles ampliaciones, CN Vandellòs II y III. En esta parte de la parcela Bonet Castellana plantea la zona de control, aparcamiento de trabajadores y visitantes y edificaciones auxiliares, como depósitos; talleres; edificio administrativo y comedor del personal (figura 21).

La propuesta de Bonet Castellana segrega el sistema viario. El trazado para vehículos, de $10 \mathrm{~m}$ de ancho, bordea perimetralmente las edificaciones y conecta las diferentes plataformas, con el fin de dar acceso rodado a las edificaciones técnicas por cuestiones de seguridad y mantenimiento. La red peatonal se resuelve en la plataforma de la cota +16 . En esta cota intermedia se disponen los accesos peatonales a todas las edificaciones: talleres; depósitos; comedor; lavanderia; vasija del reactor y edificios administrativos. De esta manera, vehículos y peatones, no comparten el mismo recorrido, ni tan solo su posición respecto los accesos de las edificaciones.

Plásticamente intentamos dar el máximo valor escultórico a las formas arquitectónicas y damos una importancia primordial al color. ${ }^{21}$

Con esta cita, Bonet Castellana inicia en 1938 la incorporación de las disciplinas plásticas en la arquitectura. En este sentido, utiliza un remate escultórico para realizar las cubiertas de todas las edificaciones. De esta manera, estructuras que albergan diversos usos, como talleres, oficinas e incluso, la vasija del reactor, tendrian un carácter unitario. La solución planteada en la central nuclear forma parte de la experimentación del arquitecto. Esta misma solución escultórica la uti- liza en la casa Cruylles (1964-68), una vivienda unifamiliar frente al mar, con la montaña en el fondo, proyecto que realiza el mismo año que el de la central nuclear (figura 22).

La propuesta de Bonet Castellana para la central nuclear incorpora el arte a partir de remates escultóricos en las cubiertas (figura 23), además de la utilización del color, como
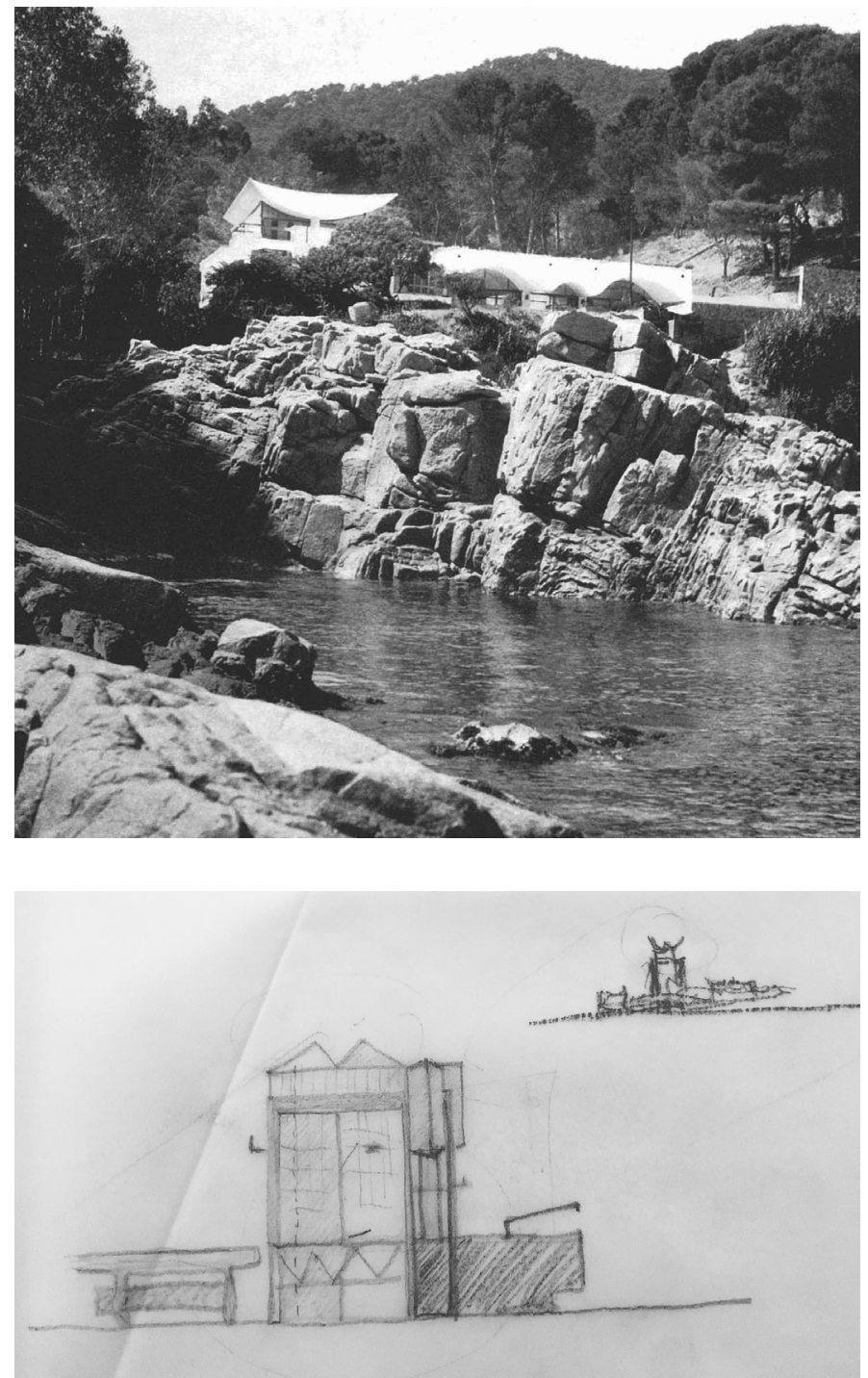

Figura 21. CN Vande1lòs. Alzado desde el acceso (primera versión). Fuente: Elaboración propia.

Figura 22. Casa Cruylles (1964-68), Antonio Bonet Castellana.

Fuente: Fons Bonet AHC.

Figura 23. CN Vandellòs. Croquis de la primera propuesta para el edificio de contención del reactor. Fuente: Fons Bonet AHC. 
Figura 24. CN Vandellòs. Almacén técnico (07/2017). Fuente:

Fotografia autor

Figura 25. CN Vandellòs. Pabellón telefónico (ca. 1972). Fuente: Fons Bonet AHC. plantea en el manifiesto Austral de 1939. En este sentido, igual que la propuesta del $\mathrm{Pa}$ lais du Gouverneur, Le Corbusier, Chandigarh (1951), el reactor de la CN Vandellòs se convertiria en un hito visual, de $50 \mathrm{~m}$ de ancho y $80 \mathrm{~m}$ de altura. Ambas propuestas, Le Corbusier en Chandigarh y Bonet Castellana en la CN Vandellòs, plantean un remate escultórico, con una solución en bóveda invertida de grandes dimensiones como hito visual en el paisaje.

EDF desestima la propuesta presentada por Bonet Castellana, tanto la parte urbanística, como la resolución plástica. En su lugar, EDF toma la central de referencia francesa de Saint Laurent des Eaux (1963-69) y se proyectan intervenciones de menor coste económico, siguiendo los principios de la empresa eléctrica y tecnológica.

Estas intervenciones incluyen la construcción de tres pabellones técnicos, control de acceso, meteorológico (figura 25) y telefónico; mejoras en la urbanización y acabados exteriores de las edificaciones de almacenes (figura 24) y vasija del reactor. En lugar de plantear una solución escultórica en las cubiertas, la nueva propuesta contempla utilizar una estrategia cromática, a partir de la construcción de un bardaje metálico de color rojo pompeyano. Esta estrategia permite unificar los criterios de las diferentes intervenciones. Además, la propuesta incorpora elementos de escala doméstica, como bancales de piedra seca en la urbanización y jardineras de 80 $\mathrm{cm}$ de altura en todas las edificaciones. Estos elementos son añadidos a los edificios proyectados por EDF que permiten acompañar en la escala humana del trabajador, resuelven el contacto con el plano del suelo y disminuyen el impacto visual de estas edificaciones de carácter industrial.

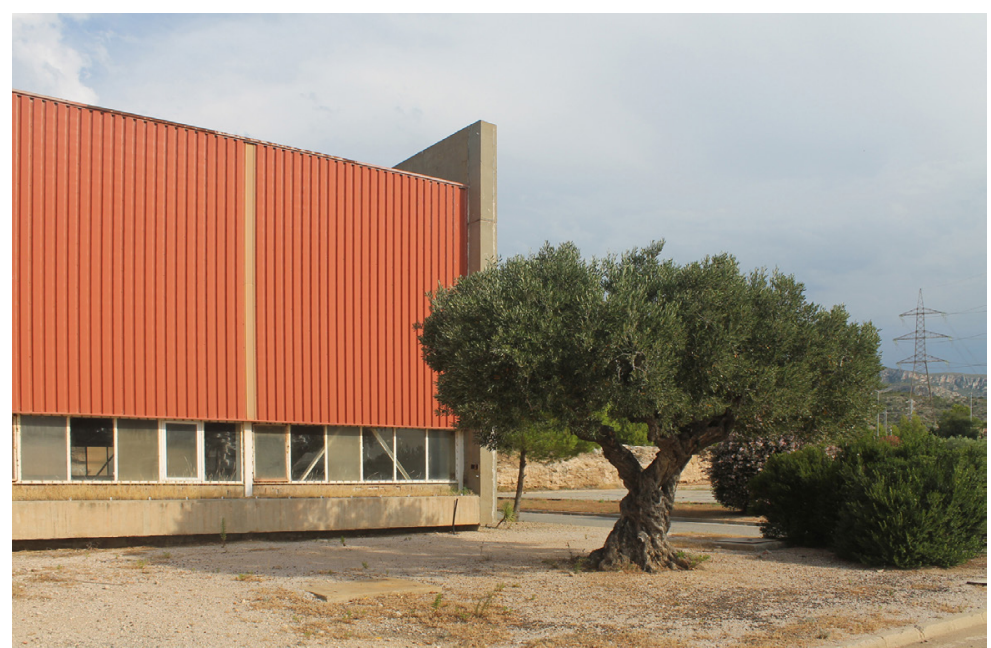

\section{Conclusiones}

La energía del átomo ha tenido una connotación negativa desde sus inicios en 1945, por su utilización militar en la II Guerra Mundial. Sin embargo, durante los años 60, el gobierno franquista utiliza esta fuente de energía para aumentar la producción eléctrica en las áreas más pobladas e industrializadas de la península.

Estas nuevas centrales nucleares son marcas visuales en el paisaje y precisan del entorno para su comprensión; bien sea para activarlo, siguiendo el modelo francés, o bien para ocultar sus estructuras, siguiendo el modelo estadounidense. La construcción de estas centrales, como apunta Claude Parent en 1974 y se ha desarrollado en este artículo, tiene una lectura a diferentes escalas: geográfica, intermedia y próxima. El trabajo de los arquitectos consiste en el desarrollo de tareas para la implantación y cómo estas centrales se perciben desde el exterior. No obstante, el arquitecto está limitado en su intervención: no puede determinar ni la colocación, ni la orientación, ni los materiales de las edificaciones principales.

En otros casos, como centrales hidroeléctricas o térmicas, el arquitecto puede desarrollar la totalidad del proyecto, desde la implantación hasta la definición completa del complejo productivo. Sin embargo, debido a las fuertes restricciones técnicas que implica la construcción de una central nuclear limita el trabajo de estos tres arquitectos. A partir de una definición previa óptima facilitada por la empresa tecnológica y de ingeniería, el arquitecto es el encargado de darle una imagen coherente y trabajar con las herramientas restantes disponibles, como acabados exteriores; volúmenes; luces; sombras; escala y ritual de aproximación.

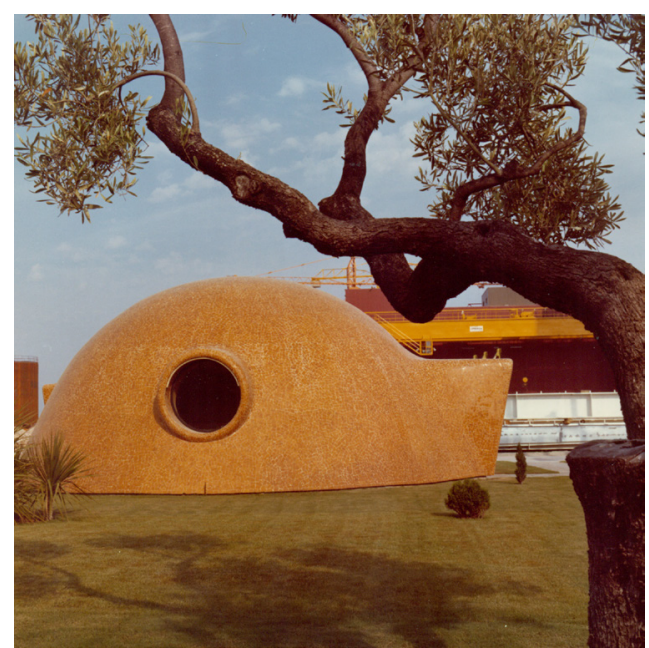


En este sentido, la intervención de Fernández Alba no se puede considerar como única, sino como la suma de diversas intervenciones requeridas por la empresa eléctrica, entre 1964 y 1968. Esta falta de unidad en la intervención interfiere en la vida cotidiana de los trabajadores, la convivencia con la escala de las máquinas de gran tonelaje y cómo esta central se percibe desde la carretera, el río y el poblado.

Por el contrario, Castelao parece haber desarrollado previamente diferentes tanteos de gran fuerza plástica para la cubrición de la vasija del reactor y la sala de turbinas ${ }^{22}$. Si observamos otros trabajos que realiza en su totalidad en las centrales hidroeléctricas de Silvón, Arbón y Las Arenas, se puede apreciar cómo trabaja, por un lado, el emplazamiento, a partir del viario, recorrido de aproximación y descubrimiento visual de las edificaciones; y por otro lado, la escala humana, a partir del material, iluminación cenital y composición de los edificios.

Finalmente, la propuesta previa de Bonet Castellana tiene una lectura clara del lugar, permite mejorar el trazado urbanístico, accediendo con una vista del mar; y considera la vasija del reactor como una escultura de grandes dimensiones. No obstante, la propuesta construida se limita a realizar edificaciones auxiliares de menor envergadura; la construcción de elementos de la urbanización, como bancales, pasos peatonales y jardineras; y dotar a la vasija del reactor y almacenes técnicos de un bardaje metálico de color rojo. En este sentido, mientras que la CN José Cabrera utiliza el naranja para camuflarse en un terreno árido, Bonet Castellana utiliza el rojo en contraposición al verde predominante en el paisaje de pinos piñoneros de la costa mediterránea. Con esta estrategia cromática permite activar el lugar con la construcción de la central nuclear.

Los requerimientos técnicos y frente la seguridad en este tipo de instalación nuclear imposibilita compatibilizar la tarea de los ingenieros con decisiones relevantes del arquitecto. No obstante, pese no haber seguido directamente las directrices planteadas por Bonet Castellana y Castelao en las centrales de Vandellòs y Santa María de Garoña, el papel del arquitecto sigue siendo necesario para desvelar los materiales; resolver armoniosamente el conjunto; segregar el sistema rodado y peatonal y cómo estas dos centrales se perciben desde la carretera, las poblaciones que las rodean, áreas residenciales con turismo (figura 18), recorrido de aproximación y poblados para trabajadores construidos en sus inmediaciones. Como conclusión final, este artículo pone en valor las tareas, tanto del equipo de arquitectos, como del equipo de ingenieros, ya que como se ha mencionado en la introducción, estas tres centrales nucleares van a ser derribadas en las próximas dos décadas.

\section{Notas}

1 España genera el 20,30\% de la electricidad consumida procedente de las 7 centrales nucleares que todavía siguen operando: Almaraz 1, Almaraz 2, Ascó 1, Ascó 2, Cofrentes, Trillo 1 y Vandellòs 2. Frente el 71,60\% en Francia o el $20,00 \%$ en Estados Unidos. Datos extraídos de la tabla "Nuclear Share of Electricity Generation in 2017" del IAEA. Fecha de consulta 15/3/2019.

2 El papel de la arquitectura de las centrales nucleares francesas y estadounidenses ha sido estudiado por Davide Di Capua y Jacinthe Pesci, con los trabajos "Le nucléaire et la Ville: Un programme pour la reconversion de la centrale nucléaire d'Indian Point" (2012) y "Domestiquer l'atome: Claude Parent et le Collège des architectes du nucléaire, une épopée française, 1974 1982" (2015).

3 Las centrales nucleares en Estados Unidos están situadas entre 40 y $100 \mathrm{~km}$ de las ciudades con población superior a 100.000 habitantes. Francia sitúa estas centrales a una distancia entre 20 y $40 \mathrm{~km}$. (Lavie y Vathaire 1967:331334).

4 Durante la I Guerra Mundial los objetivos militares en Estados Unidos son los centros de producción militar e industrial. Éstos se sitúan en las periferias de las grandes ciudades. Tras el ataque de Pearl Harbour en diciembre de 1941 se contempla la posibilidad de descentralizar las nuevas fábricas para construirlas en áreas rurales escondidas y prácticamente despobladas del sur y suroeste de Estados Unidos (Haskell 1939:70-71).

5 Claude Parent (1923-2006) es arquitecto y teórico de la fonction oblique que formula junto con Paul Virilio. Jean-Claude Lebreton, ingeniero de EDF, es quien persuade al arquitecto para trabajar en EDF. Además de la construcción de centrales nucleares, Parent publica tres libros sobre temática nuclear: L'Architecture et le nucléaire (1978), Les maisons de l'atome (1983) y Les totems de l'atome (2014).

6 Las empresas eléctricas buscan emplazamientos que reúnan las condiciones óptimas para la construcción y el funcionamiento de las centrales nucleares: situación próxima pero suficientemente alejada de los centros de consumo y redes eléctricas; caudal de agua abundante y constante para la refrigeración de la maquinaria; buenas condiciones meteorológicas, geológicas y sísmicas y buena comunicación por carretera o ferrocarril (Pascual, et al. 1967:93-105). 
7 SOM proyecta 7 centros de investigación nuclear: Laboratory of Nuclear Studies at Cornell University (1947), Brookhaven National Laboratories (1952), Los Alamos Scientific Laboratory (1952-1955), Argonne National Laboratory (1959), Radiation Laboratory at University of $\mathrm{NO}^{-}$ tre Dame (1962), University of Chicago Laboratory for Astrophysics and Space Research at the Enrico Fermi Institute for Nuclear Studies (1965) y Livermore Atomic Energy Facility (1966-1969). Gio Ponti proyecta Faculty of Nuclear Physics en Sao Paolo, Brasil (1953). Van den Broek \& Bakema proyecta el Nuclear Reactor and Institutes TU Delft (1957-1962). Philip Johnson proyecta el Soreq Nuclear Research Reactor en Soreq, Israel (1960).

8 Pedro Durán Farell (1921-1999) es ingeniero de caminos y hombre de confianza del Banco Urquijo en Cataluña. Ocupa cargos representativos en empresas como Hidroeléctrica de Cataluña, La Maquinista Terrestre y Marítima y Catalana de Gas y Electricidad. En 1960 plantea la posibilidad de llevar gas natural a Cataluña desde África. Promotor de Gas Natural S.A. y de la CN Vandellòs I.

9 Jaime McVeigh de Alfos es ingeniero industrial de la empresa Tecnatom y promotor de la CN José cabrera. Durante los años 40 trabaja en varias patentes para los trenes Talgo.

10 La revista Austral reproduce el esquema de la revista $A C$ del GATEPAC.

11 Corrales y Molezún colaboran en la central hidroeléctrica de El Grado I (1960) y presa de la Barca (1966); Fisac en la central hidroeléctrica de Canfranc (1945-69); Vaquero Palacios en las centrales hidroeléctricas de Salime (1945-56), Miranda (1956-61), Proaza (1964-68) y Tanes (1970-78) y la central térmica de Aboño (196974) y Castelao en las centrales hidroeléctricas de las Arenas (1955-58), Silvón (1956-59), Aguilar de Campoo (1962-64) y Arbón (1965-70).

12 Según la RAE, ornamentar significa adornar o engalanar con adornos algo.

13 En su archivo personal desconocen esta colaboración. Se plantea la hipótesis de que solo pudiera ser una idea de Jaime McVeigh. El único documento que se dispone de esta propuesta es una carta que envía a Antonio Fernández Alba, donde menciona "Siento mucho que al final (...) se haya torcido la ornamentación que tanto me ilusionaba hubieses hecho con Joaquín Vaquero. Realmente ha sido una lástima." Carta enviada el 9 de mayo de 1968. Legado Fernández Alba en el COAM.

14 La elección de los colores de la CN José Cabrera no es trabajo de Fernández Alba. El proyecto viene definido por la empresa tecnológica proveedora de la tecnología y del equipo de ingenieros. Mientras que los protocolos de seguridad e implantación de la AEC plantean pintar de color verde las edificaciones cuando estén próximas a zonas de vegetación frondosa, se plantea la hipótesis de que puede ser el criterio elegido para determinar el color naranja en la CN José Cabrera.

15 Juan José Elorza, ingeniero de caminos que trabajó como director de construcción para Electra de Viesgo. Colabora con Castelao en al menos cuatro centrales hidroeléctricas: Arenas de Cabrales (1955-58); Silvón (1956-59); Aguilar de Campoo (1962-64); Arbón (1965-70).

$16 \mathrm{El}$ proyecto del poblado y residencia data de julio de 1965. En la versión construida, con fecha julio de 1966, no se contempla la construcción de las 3 viviendas. Se encarga a Julio Bravo Giralt la construcción de un poblado para alojar a todas las categorias profesionales en Miranda de Ebro, con fecha junio de 1967.

17 Memoria para "Residencia y viviendas en Santa María de Garoña". Archivo Histórico de Asturias.

18 Según anotaciones personales, Castelao consulta cinco centrales nucleares en revistas de arquitectura: Estación atómica en Múnich (1959) en Informes de la Construcción; Reactor atómico en Trombay (1961) en Informes de la Construcción; Reactor Nuclear en Israel (1963) en The Architectural Review y Estación nuclear en Varsovia (1965) en Architecture d'Ajourd'hui.

19 Memoria "Edificio para fabricación de bebidas La Casera”. Archivo Histórico de Asturias

20 "El chopo será el árbol que ordene y presida paisajisticamente toda la zona". Fuente: Memoria "Residencia y viviendas de Nuclenor en Santa María de Garoña”. Archivo Histórico de Asturias.

21 Carta de Antonio Bonet a Torres Clavé (1938) (Álvarez y Roig 1999:170).

22 Consulta realizada al archivo Ignacio Álvarez Castelao (13/6/2018): "Castelao (...) como solia hacer en otras ocasiones, colabora en el diseño de la central, sirviéndose del equipo de delineantes y técnicos de Viesgo, que dibujaban según sus instrucciones casi todos los planos (...) En los planos del archivo de Castelao si está definido con su formal final el cuerpo semicilindrico y la pasarela que lo une al resto del edificio (...) Castelao propuso algo distinto para el cerramiento de la sala de turbinas y del reactor, que finalmente no fue ejecutado."

\section{Bibliografia}

Alonso, A. Inicios del programa nuclear español. Jóvenes Nucleares. Madrid, SNE.

Álvarez, F. y RoIG, J. 1999. Antonio Bonet Castellana. Barcelona: Ediciones UPC.

Bonet, A., Ferrari-Hardoy, J. y Kurchan, J. 1939. Voluntad y Acción. Austral, 1:1.

Bouvier, Y. 2005. Architecture et paysages du nucléaire: la centrale crée le site. París: Victoires éditions.

Buchanan, J.R. 1976. Siting of Nuclear facilities. Oak Ridge: NCIS.

Demaret, J. y Dufau, P. 1967. Energie nucléaire. EDF Centrales de Chinon. L'Architecture d'Ajourd'hui, 133:32-34.

Drexler, A. 1969. Twentieth Century Engineering. Nueva York: Museum of Modern Art.

García-Pola, M. A. 1997. Astúries: L'èpica del desenvolupament. Quaderns d'Arquitectura $i$ Urbanisme, 215:92-99. 
Gonzalvo, C., Ródenas, J.F. y Zuaznabar, G. 2018. Centrales nucleares y patrimonio, el caso de la central nuclear de Vandellòs I (Tarragona). E-RPH Revista electrónica de Patrimonio Histórico, 23:1-23.

Haskell, D. 1939. What does military design offer the planning of peace. Architectural Record, 3:68:75.

IBERDUERO. 1971. Nuclenor. Santander: Iberduero.

Lavie, J M. y Vathaire, F. 1967. Contribution a l'etude de l'implantation des centrales nucléaires aupres des villes. En: VV. AA., Containment and siting of nuclear power plants. Viena: International Atomic Energy Agency. 331-342.

Parent, C. 1989. L'impact esthétique des centrales. Aménegement et Nature, 94: 5.

Pascual, F., Alonso, A., Noreña, S. y Sevilla, A. 1967 Los emplazamientos de las primeras centrales nucleares españolas. En: VV.AA., Containment and siting of nuclear power plants. Viena: International Atomic Energy Agency. 93-104.

Thompson, E. 1957. For an Architecture of Nuclear Buildings. Architectural Record, 121:182.

VAN DER RoHE, M. 1950. Architecture and technology. Arts \& Architecture, 10:30.

VAQUeRo, J. 1998. La integración de las artes: ideales, trabajos y recuerdos de mi obra en las centrales. En: VV.AA.: Vaquero Palacios (19001998). Madrid: Consejo Superior de los Colegios de Arquitectura de España. 116.

VV. AA. 1971. Central Nuclear de Santa María de Garoña. ROP Revista de Obras Públicas, 3079:852.

Yadigarogly, G y Andersen, A. 1976. Novel siting solutions for Nuclear Power Plants. En: Buchanan, J.R. Siting of Nuclear Facilities. Oak Ridge: NSIC. 